University of Nebraska - Lincoln

DigitalCommons@University of Nebraska - Lincoln

USDA Forest Service / UNL Faculty Publications U.S. Department of Agriculture: Forest Service --

National Agroforestry Center

2000

Direct and indirect parametrization of a localized model for the mountain pine beetle - lodgepole pine system

\author{
Zy Biesinger \\ Utah State University \\ James Powell \\ Utah State University \\ Barbara Bentz \\ Utah State University \\ Jesse Logan \\ Utah State University
}

Follow this and additional works at: https://digitalcommons.unl.edu/usdafsfacpub

Part of the Forest Sciences Commons

Biesinger, Zy; Powell, James; Bentz, Barbara; and Logan, Jesse, "Direct and indirect parametrization of a localized model for the mountain pine beetle - lodgepole pine system" (2000). USDA Forest Service / UNL Faculty Publications. 84.

https://digitalcommons.unl.edu/usdafsfacpub/84

This Article is brought to you for free and open access by the U.S. Department of Agriculture: Forest Service -National Agroforestry Center at DigitalCommons@University of Nebraska - Lincoln. It has been accepted for inclusion in USDA Forest Service / UNL Faculty Publications by an authorized administrator of DigitalCommons@University of Nebraska - Lincoln. 


\title{
Direct and indirect parametrization of a localized model for the mountain pine beetle — lodgepole pine system
}

\author{
Zy Biesinger ${ }^{\mathrm{a}, *}$, James Powell ${ }^{\mathrm{a}}$, Barbara Bentz ${ }^{\mathrm{b}}$, Jesse Logan ${ }^{\mathrm{b}}$ \\ ${ }^{a}$ Department of Mathematics and Statistics, Utah State University, Logan, UT 84322-3900, USA \\ ${ }^{\mathrm{b}}$ USDA Forest Service Rocky Mountain Research Station,
}

Logan Forestry Sciences Laboratory and Department of Forest Resources, Utah State University, Logan, UT 84322-3900, USA

Received 21 May 1999; received in revised form 16 December 1999; accepted 7 February 2000

\begin{abstract}
The dynamic interaction between mountain pine beetles (MPB) and one of its hosts is reviewed briefly. The 'local' projection of a partial differential equation model describing this interaction is employed in model parameter estimation. Methods and assumptions for estimating non-fitted parameter values are given. Assigning values to non-fitted parameters, direct and indirect parametrization techniques are employed to estimate remaining parameter values. The indirect method is quickly and easily applied to many data sets but requires some assumptions and model simplifications. The direct method requires fewer assumptions but is computationally intensive. The results of these two techniques are compared and evaluated. (C) 2000 Elsevier Science B.V. All rights reserved.
\end{abstract}

Keywords: Bark beetles; Parameterization; Spatial models

\section{Introduction}

The spatial redistribution of organisms in an ecosystem is often a complex process. Many ecosystems exhibit spatial complexity on a broad range of scales, driven by the physical environment and biotic interactions (Roberts, 1987). As different species aggregate and disperse in spatially complex ways, even an initially uniform environment can soon evolve spatial heterogeneity. There is a variety of factors that drive the spatial dynamics of a population, including de-

\footnotetext{
* Corresponding author. Tel.: + 1-435-7972485.

E-mail address: zy@biology.usu.edu (Z. Biesinger).
}

fense against predators, success as predators, and reproductive success. Spatial complexity can also arise from dispersal and aggregation. When an organism is itself responsible for chemical, auditory, visual or other cues which lead to aggregation, non-linear feedback can occur, creating self-organized groups of individuals (aggregation). Dispersal also plays an important role in many ecosystems' dynamics. Interactions between aggregative and dispersive forces lead to complex spatial reorganization of populations of organisms.

Spatial dynamics typically play a central role in the community dynamics of highly mobile insects (Turchin and Thoeny, 1993), such as mountain 
pine beetle (MPB, Dendroctonus ponderosae Hopkins) (Preisler and Haiganoush, 1993). The MPB/ pine tree host (e.g. Pinus contorta) system has long been the subject of research because of the economic and ecological impact of the beetles. As an aggressive bark beetle (one that kills its host), eruptions of this species are impressive events resulting in intensive and extensive outbreaks. It is also becoming recognized that disturbances, such as insect outbreaks, may be central to maintaining the structure, function and health of western forests (Roe and Amman, 1970; Mattson and Addy, 1975; Schowalter et al., 1981; Romme et al., 1986).

One method to help understand this dual role of bark beetles as an economic competitor and as a co-evolved component of the ecosystem is the development and analysis of quantitative models (Berryman, 1976, 1982; Burnell, 1977; Berryman et al., 1984, 1989; Raffa and Berryman, 1986; Safranyik et al., 1989; Turchin, 1989; Polymenopoulos and Long, 1990; Turchin and Thoeny, 1993). These spatially independent models have achieved some success, but fail to capture the full sequence of events necessary for successful population establishment and expansion. Aggregation on and dispersal from a host are of such overriding importance to MPB ecology that including spatial dynamics in model representations is essential for ecological credibility.

The theoretical literature abounds with models for the tactic interactions of species with themselves, one another, or their resource environment (see, for example, reviews in Murray, 1989; Holmes et al., 1994; Turchin and Simmons, 1998). However, the penetration of theoretical viewpoints into the real world of spatial ecology is limited by the lack of parametrized spatial models. Whereas many models of purely temporal population phenomena have been parametrized and subsequently used to organize understandings in practical population ecology (see, for example, Gause, 1934; Harrison, 1995; Ellner and Turchin 1995; Haefner, 1996), input from the world of PDE models of spatial dynamics is still mainly in the form of hints and possibilities but not actualities. Of course, parametrizing a spatial model is no simple task. The mass of data needed for spatio-temporal systems is daunting, and often simply too expensive to collect for many systems of interest. Then, almost all solutions to PDE of ecological interest involve parameters non-linearly (see, for example, Turchin and Thoeny, 1993), necessitating non-linear least-squares procedures on large data sets with complicated and often poorly-behaved functions.

The situation becomes worse when parametrizing models which include taxis. The non-linearity of these models means that almost no appropriate analytic solutions can be found, and consequently the non-linear least-squares procedure must itself be based on numerical solutions to spatially extended equations. An alternative is to use fluxbased procedures (as in Turchin and Simmons, 1997, 1998), which hinge on being able to measure the fluxes of organisms (which we were repeatedly unable to do in the MPB/host system, and which is probably problematic with any population composed of organisms capable of avoiding flux traps or sticky panels).

The current manuscript addresses these general problems by utilizing a 'local projection' (Powell and Rose, 1997) to parametrize a PDE-based model. On the one hand, data requirements are simplified to time series at isolated points in space, which simplifies both the observational and computational difficulties. On the other hand, the localization frames the model as an ordinary differential equation at a point rather than an extended PDE, simplifying procedural and numerical aspects of the regression problem. Altogether, parametrization is rendered practically and computationally realistic by our approach. The procedures are illustrated using the Powell et al. (1996) model for MPB/host interactions, but should be feasible for a variety of spatial models describing plant/animal interactions.

\subsection{System description and behaviour}

During a relatively short flight period in late summer, adult MPB fly in search of suitable host trees into which females deposit eggs. Throughout the next year juvenile MPB move horizontally through the phloem tissue, feeding on the energy rich products of photosynthesis. Pine tree hosts 
do not passively submit to MPB attacks, but have evolved physiological mechanisms to resist them (Smith, 1963, 1966; Reid et al., 1967; Nebeker et al., 1993; Raffa et al., 1993). Healthy and vigourous trees may resist beetle attacks by flooding the gallery with resin which serves as a chemical and physical barrier to incoming beetles.

Only those trees with a rapid and sustained reaction are likely to survive an MPB mass attack (Berryman et al., 1989; Raffa et al., 1993), therefore, beetles must synchronize their emergence and coordinate their attacks on a specific host tree quickly enough to deplete the resin reserves (Amman and Cole, 1983). To coordinate attacks, MPB have evolved a chemical communication system (Pitman et al., 1968; Pitman, 1971; Hughes, 1973) based on diffusable aggregation (McCambridge, 1967; Geiszler and Gara, 1978; Geiszler et al., 1980; Conn et al., 1983; Borden et al., 1987) and anti-aggregation (Rudinsky et al., 1974; Ryker and Yandell, 1983; Libbey et al., 1985; Hunt et al., 1989) pheromones and kairomones (Renwick and Vite, 1970; Roe and Amman, 1970; Moeck and Simmons, 1991).

Aggregation feedback has the potential to attract large numbers of MPB. Over-colonization is avoided via an anti-aggregation arm of the pheromone communication system. In addition to aggregants, attacking males and females release a suite of compounds resulting in a close range redirection of responding beetles to nearby trees (McCambridge, 1967; Geiszler et al., 1980; Borden et al., 1987; Bentz et al., 1996). This chemical communication system allows beetle populations to survive from year to year at endemic levels, killing only weakened trees. A population is considered endemic when there are only one or two infested trees scattered across 2 hectares. As population numbers grow, more vigourous and more nutritional trees can be overcome. If there are more than two clumps of at least four to five infested trees over 2 hectares, then the population is considered to be building to the epidemic phase and can eventually result in thousands of hectares of killed trees.

\subsection{Global model}

The mass dispersal behaviour of the MPB/host tree system is described by a model (Powell et al., 1996; Powell and Rose, 1997; Powell et al., 1997a,b, 1998) using density variables (number or amount per hectare) depending on spatial location, $x, y$, and time, $t$. The state variables are:

$Q(x, y, t)$ - population of MPB attacking susceptible trees

$H(x, y, t)$ - number of entrance holes bored by attacking MPB

$S(x, y, t)$ - resin outflow

$R(x, y, t)$ - resin capacity of initially uninfested trees

$P(x, y, t)$ - population of MPB dispersing from previous year's infested trees

$A(x, y, t)$ - concentration of pheromones

The model equations are:

$$
\begin{aligned}
& \dot{Q}=-\omega_{2} Q+r_{1} \frac{R}{R_{0}} P-\beta S \frac{Q}{H} \\
& \dot{H}=r_{1} \frac{R}{R_{0}} P-r_{4} r_{3} H R \\
& S=r_{3} H R \\
& \dot{R}=\left[r_{2}\left(R_{0}-R\right)-r_{3} H\right] R \\
& \frac{\partial}{\partial t} P=-\nabla \cdot\{[v \nabla f(A)] P-\mu \nabla P\}-\omega_{1} P \\
& \quad-r_{1} \frac{R}{R_{0}} P+\gamma
\end{aligned}
$$

where

$f(A)=A_{3} A_{0}\left\{\left(A_{3}+1\right) \ln \left[1+\frac{A}{A_{3} A_{0}}\right]-\frac{A}{A_{0}}\right\}$

and

$\frac{\partial}{\partial t} A=b_{1} \nabla^{2} A+a_{1} Q-\delta_{1} A$

\subsection{Localization of the global model}

The global model, describing dynamics on a forest-sized scale, has proven to be too complicated for ecological use, because variables describing an entire forest are spatially and temporally 
extended. Localizing the global model will convert variables from densities to numbers at a point. It is these types of numbers that field studies generate. This allows the use of observational data to approximate parameter values which best fit the local model to observed system dynamics. To localize the model we investigate the consequences of the global model at a single location (for example, an individual host) (Powell et al., 1996). Local model variables depend only on time, $t$ :

$q(t)$ - population of (alive) nesting MPB in a tree

$h(t)$ - number of open attack holes in a tree

$r(t)$ - resin capacity of a tree

$a(t)$ - number of pheromone molecules at a point

$w_{a}(t)$ - characteristic area occupied by the pheromone plume from a single host

$I(t)$ - local population of dispersing MPB

With these variables we construct the local model following the localization method described in Powell et al. (1996) and Powell and Rose (1997), yielding equations:

$$
\begin{aligned}
& \dot{q}=r_{1} \frac{r}{r_{0}} I-\beta \frac{r_{3}}{w} q r \\
& \dot{h}=r_{1} \frac{r}{r_{0}} I-\frac{r_{3}}{w} r_{4} h r \\
& \dot{r}=r\left[\frac{r_{2}}{w}\left(r_{0}-r\right)-\frac{r_{3}}{w} h\right] \\
& \dot{a}=a_{1} q-\delta_{1} a \\
& \dot{w}_{a}=4 b_{1}+a_{1} q \frac{w-w_{a}}{a}
\end{aligned}
$$

and

$$
I=\frac{\pi \gamma r_{e}^{2}}{r_{1}+\omega_{1}} \exp \left[\frac{v}{\mu} f(q)\right]
$$

where

$$
\begin{aligned}
f(q)= & A_{3} A_{0}\left\{\left(A_{3}+1\right) \ln \left[1+\frac{2 a_{1} q}{A_{3} A_{0}\left(4 b_{1}+w \delta_{1}\right)}\right]\right. \\
& \left.-\frac{2 a_{1} q}{A_{0}\left(4 b_{1}+w \delta_{1}\right)}\right\}
\end{aligned}
$$

A list of parameter definitions is presented in Table 1.

\section{Methods}

\subsection{Experimental design and data collection}

During the summer of 1995, three sites, at least $400 \mathrm{~m}$ apart, were selected in a lodgepole pine stand in the Gold Creek drainage of the Sawtooth National Recreation Area (SNRA), ID. One MPB pheromone bait (Phero Tech Inc., Delta, $\mathrm{BC}$, Canada) was attached to a single focus tree at each site and removed after $24 \mathrm{~h}$. Based on emergence data from a nearby experiment (Bentz, 1995) beetle flight in the area had just begun. For twelve days MPB attacks on the basal $2.1 \mathrm{~m}$ of individual trees were counted twice a day on all host trees within $10 \mathrm{~m}$ of the focus tree. By monitoring MPB attacks on individual trees we were able to create a time series of the landing and attack of recently emerged beetles (Bentz et al., 1996) (Figs. 1 and 2). Due to lack of attacked trees in two of the plots, only results from successfully attacked trees in one plot are reported.

Two similar sites were established in 1996 near St. Charles, ID. In 1997, five plots were monitored, two new sites near St. Charles and three in the Williams Creek drainage in the SNRA. The focus tree of each plot was artificially baited to initiate mass attacks in the plots and the baits were removed once attacks had begun. All host trees within a radial distance of $14 \mathrm{~m}$ were monitored twice a day. MPB attacks were measured between 0.7 and $1.75 \mathrm{~m}$ from the base of each tree. Relative to temperatures during the 1995 experiments, temperatures fluctuated widely during the 1996 and 1997 experiments. Fig. 2 depicts an attack series that does not conform to expected system behaviour of a unimodal attack beginning quickly and tapering off slowly. The fluctuations in the attack series were likely to have been influenced by corresponding fluctuations in temperature.

To gather data for the purpose of validation, several weeks after beetle flight had ceased in the 1997 St. Charles area we returned to estimate the final number of beetles nesting in successfully attacked trees. We took counts of all attacks in $0.1225 \mathrm{~m}^{2}$ on the north and south aspect of each tree at heights of $0,1.4,2.8,4.2$ and $5.6 \mathrm{~m}$. Also, 
Table 1

List of parameters appearing in the global PDE and local ODE models for MPB redistribution ${ }^{\mathrm{a}}$

Parameter definitions and units

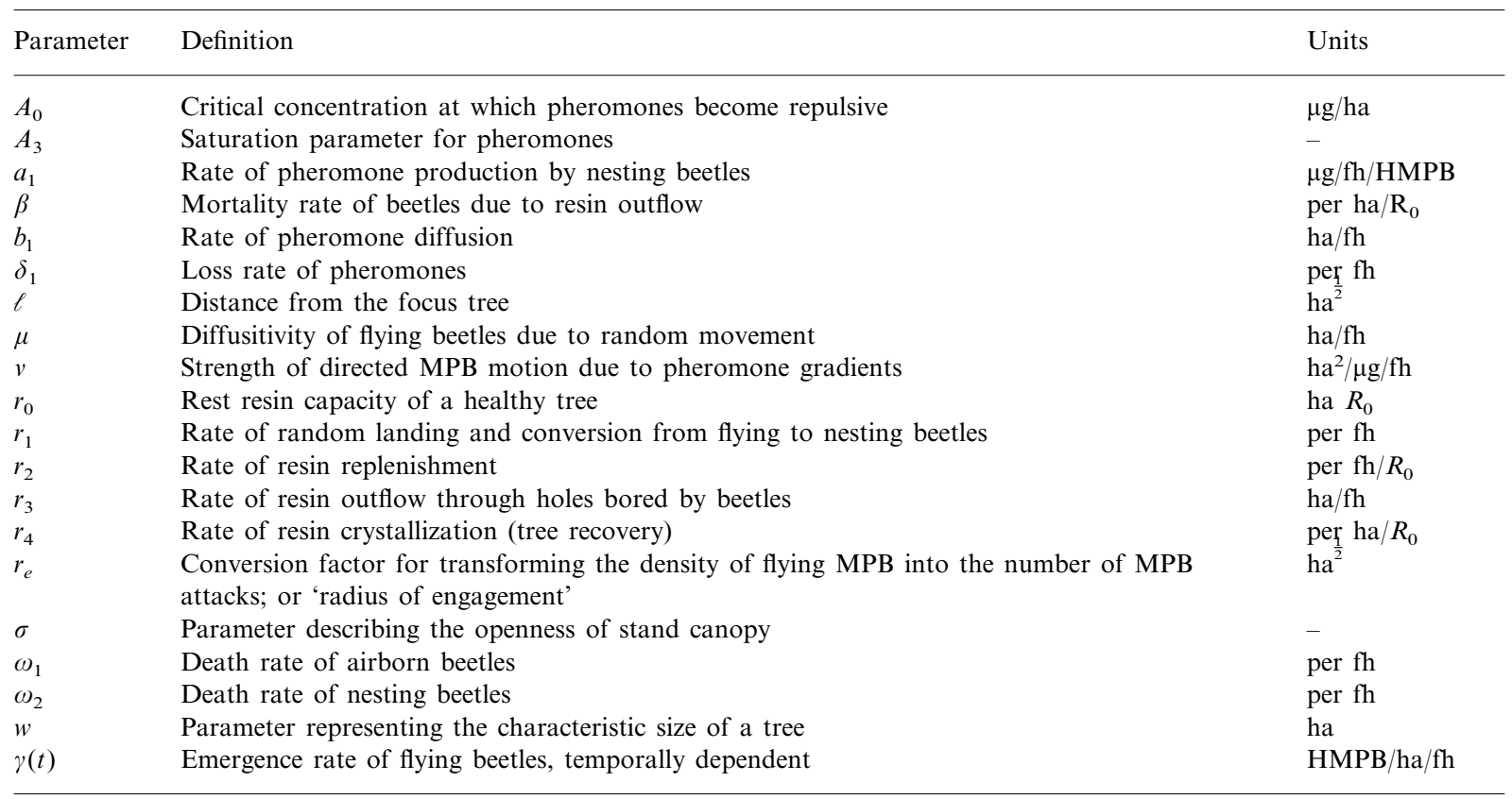

${ }^{a}$ Density units are presented with respect to hectares (ha), pheromone amounts in micrograms $\left(\mu \mathrm{g}=10^{-6} \mathrm{~g}\right.$ ), and numbers of MPB are counted in hundreds (HMPB). The basic unit of time is the flight hour (fh), of which there are approximately five per day.

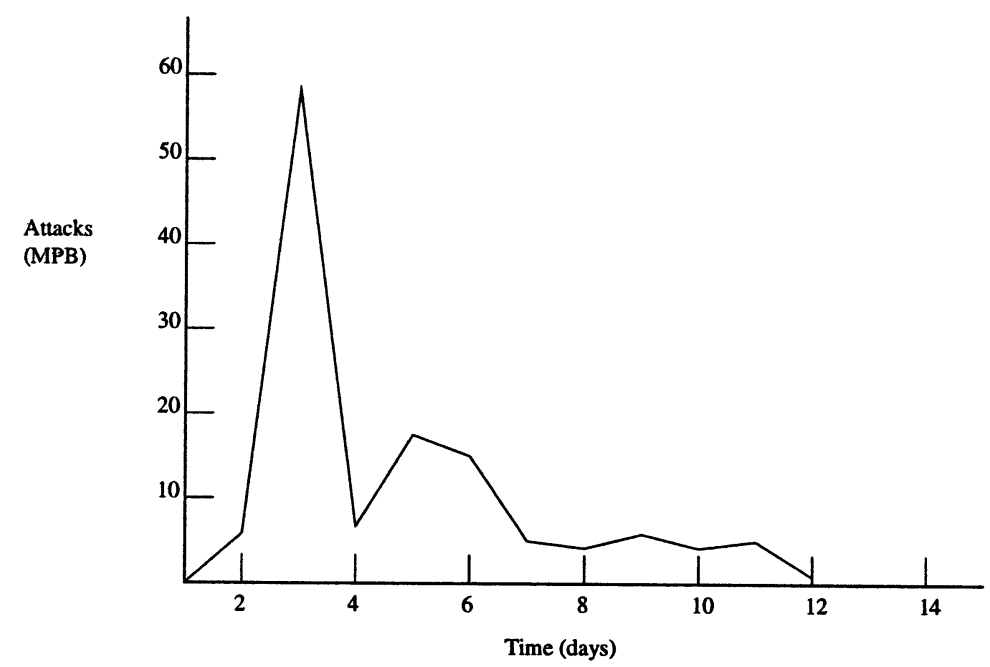

Fig. 1. Example of MPB attack series on a single host which exhibits expected system dynamics: a rapid increase in the number of attacks followed by a sharp drop. This figure shows MPB attacks on the basal $2.1 \mathrm{~m}$ of the bole of tree 3 from the 1995 SNRA data. 


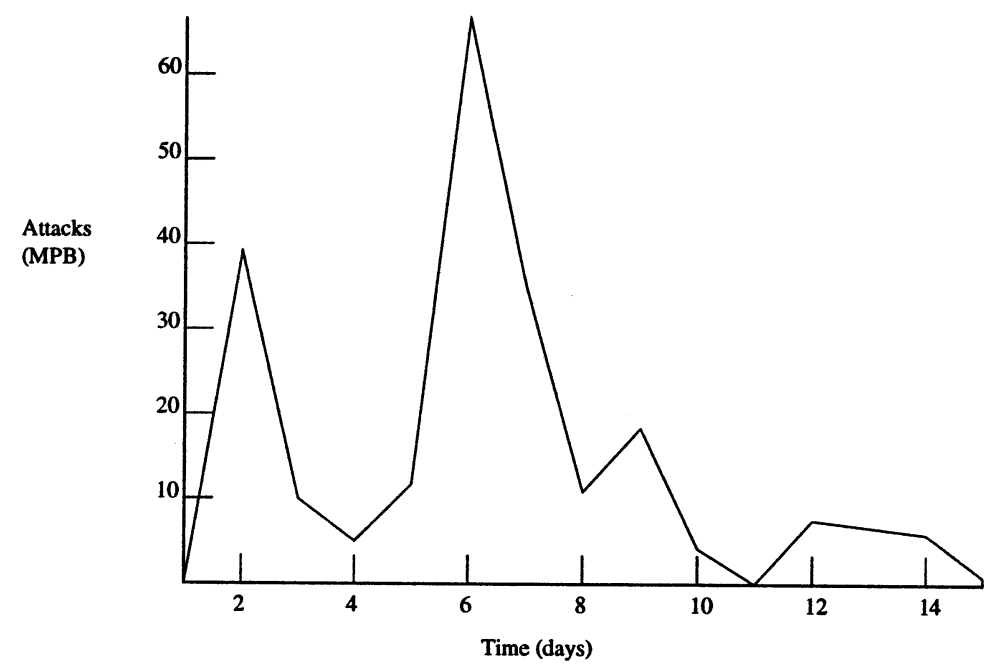

Fig. 2. Example of MPB attack series on a single host which does not exhibit expected system dynamics: multiple large peaks in attack numbers. This figure represents MPB attacks on $1.05 \mathrm{~m}$ of the bole of tree 2 in plot 2 from the 1997 St. Charles data.

we estimated the total height of attack on each tree. In parametrizing the model we assumed the maximum attack height to be $7.3 \mathrm{~m}$ (Klein et al., 1978; Raffa and Berryman, 1983), although in reality this number varied depending on the size of a particular tree.

\subsection{Local parameter values}

The parameters to be estimated fall in two catagories: 'behavioural' parameters $\left(v, \mu\right.$, and $\left.A_{0}\right)$ controlling MPB dispersal and 'local' parameters for the MPB attack/host response interaction, which can, in principle, be measured purely on a tree-by-tree basis. Here our focus is on the 'behavioural' parameters; for the local parameters we will use estimates based on information from the literature and personal experience. A survey of these estimates is presented as an appendix and closely follows those presented in Powell et al. (1996), Powell and Rose (1997) and Logan et al. (1998). In addition, we use the behavioural parameter for random dispersal, $\mu$, estimated for the southern pine beetle (Dendroctonous frontalis) (Turchin and Thoeny, 1993). Parameter values thus determined are presented in Table 2.

The remaining two behavioural parameters, $A_{0}$ and $v$, are difficult to estimate from previous information and data. The concentration $A_{0}$ describes the critical pheromone level at which beetles redirect their attack to a new tree, and $v$ describes the speed at which the MPB population responds to a given pheromone gradient. Values for $A_{0}$ and $v$ can be approximated by fitting model predictions to field data. Also, $\gamma(t)$, the emergence rate, will be left free, since it potentially varies in magnitude and shape from site to site. Two sepa-

Table 2

List of parameter values estimated using literature and knowledge of the systems involved ${ }^{\mathrm{a}}$

Non-fitted parameter values

\begin{tabular}{llll}
\hline Parameter & Value & Parameter & Value \\
\hline$A_{3}$ & 1 & $a_{1}$ & 2 \\
& & & $\mu \mathrm{g} / \mathrm{fh} / \mathrm{HMPB}$ \\
$b_{1}$ & $0.324 / \sigma \mathrm{ha} / \mathrm{fh}$ & $\beta$ & $8 \mathrm{per} \mathrm{ha} / R_{0}$ \\
$\delta_{1}$ & $360 \sigma$ per fh & $\mu$ & $1.0 \mathrm{ha} / \mathrm{fh}$ \\
$r_{0}$ & $1 \mathrm{ha} R_{0}$ & $r_{1}$ & $0.16 \mathrm{per} \mathrm{fh}$ \\
$r_{2} / w$ & $0.1 \mathrm{per}$ & $r_{3} / w$ & $0.05 \mathrm{per} \mathrm{fh}$ \\
& ha/fh $/ R_{0}$ & & \\
$r_{4}$ & 2 per ha $/ R_{0}$ & $r_{e}$ & $2 \mathrm{~m}$ \\
$\sigma$ & 0.5 & $w$ & $3 \mathrm{~m}^{2}$ \\
\hline
\end{tabular}

${ }^{\text {a }}$ Units involving resin are measured relative to $R_{0}$. Other units are: $\mu \mathrm{g}\left(10^{-6}\right.$ grams $)$, ha $\left(10^{4}\right.$ square metres $=$ hectares $)$, fh (flight hours $\sim 5 \mathrm{fh} /$ day). 
rate fitting methods, each with its own advantages and disadvantages, will be used to estimate these parameters.

\subsection{Model parametrization}

Initially we use an indirect fitting technique which can be quickly and easily applied to the MPB attack series of many trees. This technique is computationally rapid, but requires several simplifications of the local model and assumptions about system conditions which increase error propogation. To check the effectiveness and robustness of the indirect method, we fit the model via non-linear least-squares directly to the same attack series. Although the direct fitting technique requires much more time than the indirect method, it requires fewer assumptions.

\subsubsection{Indirect fitting method}

The indirect fitting method is based on the assumption that the number of new MPB attacks on a tree largely depends on the number of beetles already nesting in that tree. Making the assumption that the field collected data reflect the rate of attack by MPB on individual trees, the number of new attacks, $A_{n}$ corresponds to the infestation term appearing in Eqs. (6) and (7):

$r_{1} \frac{r}{r_{0}} I$

We can use this assumption to estimate $A_{0}$ and $v$, which are embedded in the infestation function, $I$ (Eq. (11)):

$$
\begin{aligned}
I= & \frac{\pi \gamma r_{e}^{2}}{r_{1}+\omega_{1}} \exp \left[\frac { v } { \mu } \left\{A _ { 3 } A _ { 0 } \left(\left(A_{3}+1\right)\right.\right.\right. \\
& \ln \left[1+\frac{2 a_{1} q}{A_{3} A_{0}\left(4 b_{1}+\omega \delta_{1}\right)}\right] \\
& \left.\left.\left.-\frac{2 a_{1} q}{A_{0}\left(4 b_{1}+\omega \delta_{1}\right)}\right)\right\}\right]
\end{aligned}
$$

By replacing the infestation term in Eqs. (6) and (7) with the field data, $A_{n}$, we use a stepwise approximation of the local model and calculate the number of nesting beetles required for the model to predict the number of new attacks actually observed. This generates ordered pairs con- sisting of the number of nesting beetles required to attract an observed number of new attacks (the dependent variable) and the number of new attacks (the independent, observed variable), $\left\{q_{n}, A_{n}\right\}$. Using a non-linear least-squares fitting technique, we can then estimate $A_{0}$ and $v$ by fitting the function $r_{1}[r(q)] /\left[r_{0}\right] I(q)$ to the data.

Since the infestation function is exponential and logarithmic (Eq. (11)) it is very sensitive to changes in the value of $q$. We manipulate the correspondence of:

$A_{n} \sim r_{1} \frac{r_{n}}{r_{0}} I\left(q_{n}\right)$

and apply a logarithmic transformation to make the infestation function less sensitive. Thus, the goal is to choose $v, A_{0}$, and $\gamma$ to maximize the correspondence between:

$\left\{q_{n}, \ln \left[\frac{A_{n} r_{0}}{r_{1} r_{n}}\right]\right\} \quad$ and $\quad\left\{q_{n}, \ln \left[I\left(q_{n}\right)\right]\right\}$

Leaving $A_{0}$ and $v$ free and assigning values to the other parameters leaves only the function describing the emergence rate of adult beetles, $\gamma(t)$, to be defined. In principle $\gamma$ is a directly measurable function, the density of MPB emerging in the 'background' of the plot. In practice it can vary extremely with temperature, shifts of wind, and spatio-temporal fluctuations in the emergence of MPB from host trees in the vicinity. Collecting these data was well beyond the scope of our study. Instead, from the local perspective, $\gamma(t)$ is just the temporal attack 'forcing' perceived within a plot, and therefore a stepwise approximation to $\gamma(t) \doteq \gamma\left(t_{n}\right)=\gamma_{n}$ is:

$\gamma_{n}=\Gamma \sum_{j=1}^{\text {all trees }}(\text { attacks on tree } j)_{n}$

where $n$ is a time index and $\Gamma$ is a scaling factor between attacks in a plot and beetle activity in the area. When solving model equations we replace $\gamma(t)$ with the scaled interpolated function, $\gamma_{n}$. Fig. 3 provides an example of the shape of the interpolated function using the counted attacks from plot 3 of the 1997 SNRA field sites.

The 'observations' $q_{n}$ and $r_{n}$ must still be estimated. Assuming the resin capacity, $r$, does not equal zero (the case where the tree is dead) and 


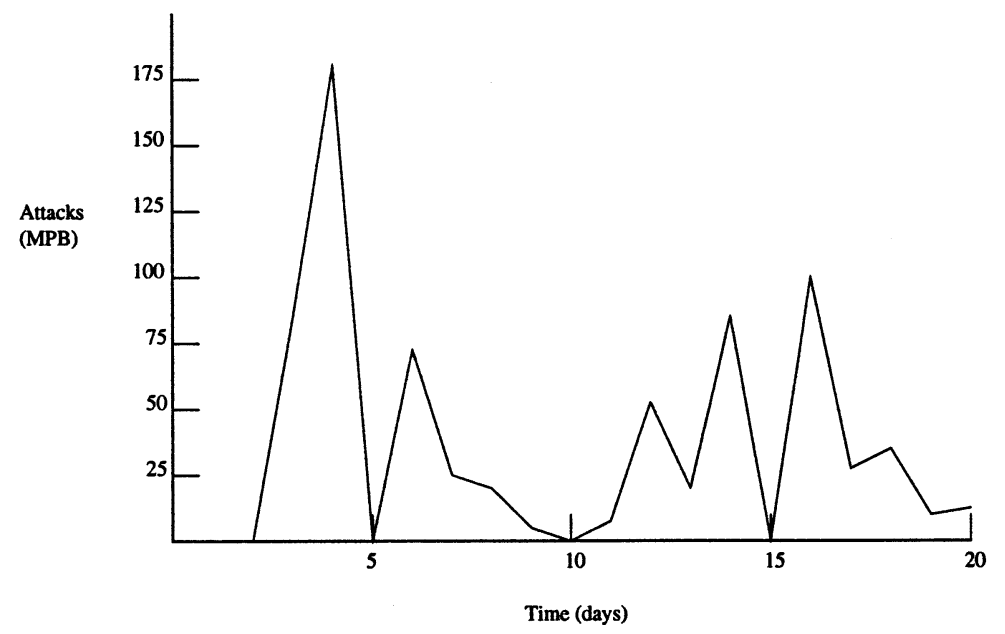

Fig. 3. Total daily MPB attacks on $1.05 \mathrm{~m}$ of tree boles in the entire 1997 SNRA plot 3, during the summer flight period. This data can be used to provide the shape of the function describing background emergence, $\gamma$, including variations of temperature and emergence non-pararnetrically.

that, on our time scale, the change in tree vigour, $\dot{r}$, is small, from Eq. (8) we have:

$r=r_{0}-\frac{r_{3}}{r_{2}} h$

In Eq. (7), taking the rate of resin crystallization, $r_{4}$, to be small on the time scale of beetle activity gives:

$\dot{h}=r_{1} \frac{r}{r_{0}} I$

Hence:

$h_{n}=$ sum of attacks observed from time 1 to $n$ $=\sum_{i=1}^{n} A_{i}$

$r_{n}=$ initial resin capacity of a tree - resin loss due to attack holes

$=r_{0}-\left[\left(r_{3}\right) /\left(r_{2}\right)\right] h_{n}$

$\dot{q}_{n}=$ rate of new attacks - rate at which beetles are repelled by tree defenses $=\left[\left(A_{n}\right) /(\Delta t)\right]-\beta r_{3} q_{n} r_{n}$

One example of $h_{n}$ appears in Fig. 4. In the equation for $\dot{q}_{n}$ we can assume that $A_{n}$ and $r_{n}$ are constant at a given time step. For $\Delta t$ use the interval between observations, and use $q_{n-1}$ as the initial condition for $q_{n}$. Solving for $q_{n}$, assuming $r_{n}$ and $A_{n}$ constant over the time interval, we have:

$$
\begin{aligned}
q_{n}= & \frac{A_{n}}{\beta r_{3} r_{n} \Delta t}\left[1-\exp \left(-\beta r_{3} r_{n} \Delta t\right)\right. \\
& \left.+q_{n-1} \exp \left(-\beta r_{3} r_{n} \Delta t\right)\right]
\end{aligned}
$$

With this stepwise model we can use the number of observed attacks in a tree and estimate the number of beetles nesting at the previous time step, thus providing the 'independent' variable for the fitting procedure. We employ a least-squares regression method to fit model predictions to observational data (Fig. 5). Specifically, we use Mathematica's Non-linearRegress routine (Martin, 1996), which uses the Levenberg-Marquardt method, gradually shifting the search from steepest descent to quadratic minimization.

\subsubsection{Direct fitting method}

The 'direct' fitting approach involves guessing parameters $A_{0}, v$, and $\Gamma$, solving Eqs. (6)-(11) numerically, and evaluating the error by summing the absolute deviation between model predictions for $r_{1}\left[r / r_{0}\right] I$ and $A_{n}$. Parameters which minimize the sum of absolute deviation are deemed 'best' fits. The direct algorithirn is summarized in pseudo-code below: 


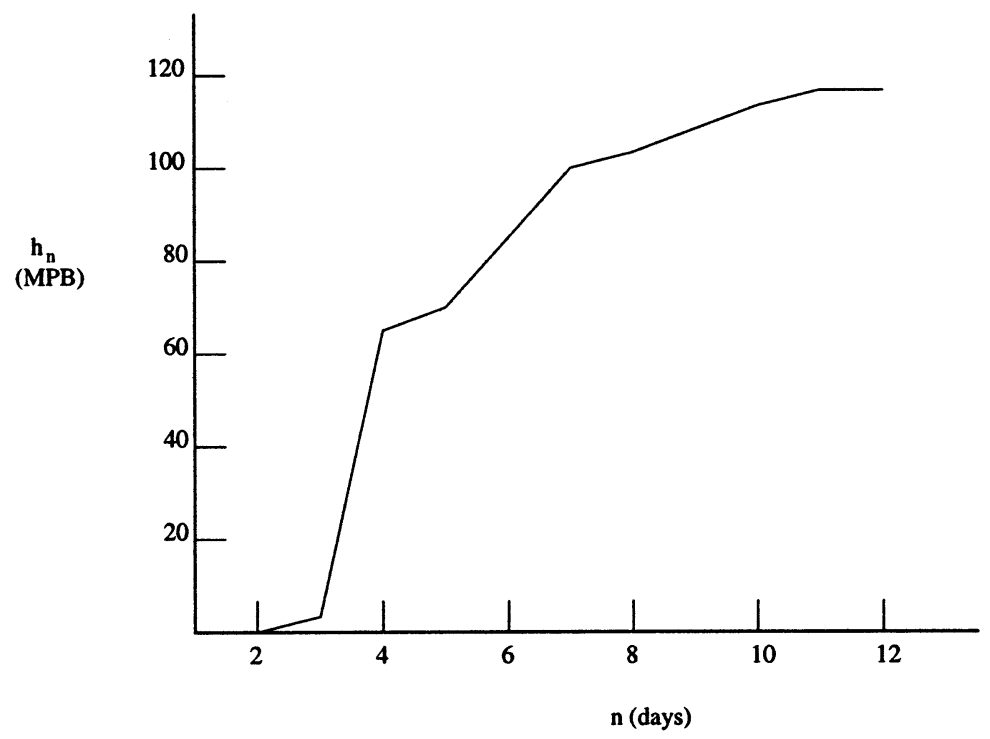

Fig. 4. Representation of all MPB attacks, $h_{n}$, on the basal $2.1 \mathrm{~m}$ of a single host up to day Eq. (12). This figure depicts the summed attacks for tree 3 from the 1995 SNRA data.

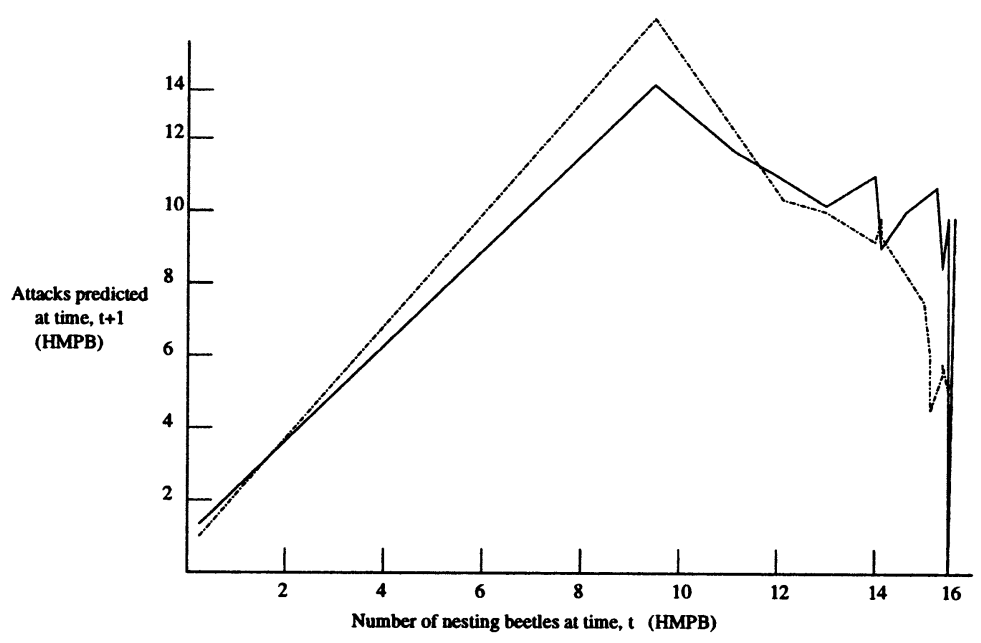

Fig. 5. Comparison of manipulated experimental data $\left(\left\{q_{n}, \ln \left(\left(A_{n} r_{0}\right) /\left(r_{1} r_{n}\right)\right)\right\}\right.$, solid line) and model predictions $\left(\left\{q_{n}\right.\right.$, ln $\left.\left(I\left(q_{n}\right)\right)\right\}$, dashed line) for MPB attacks on an entire tree using the indirect fitting procedure and the attack series for tree 5 from the 1997 St. Charles plot 1 data.

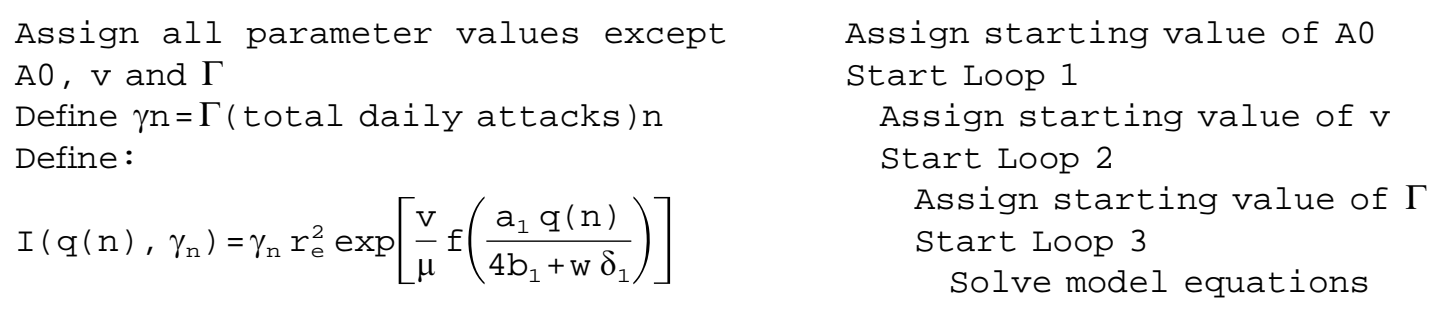




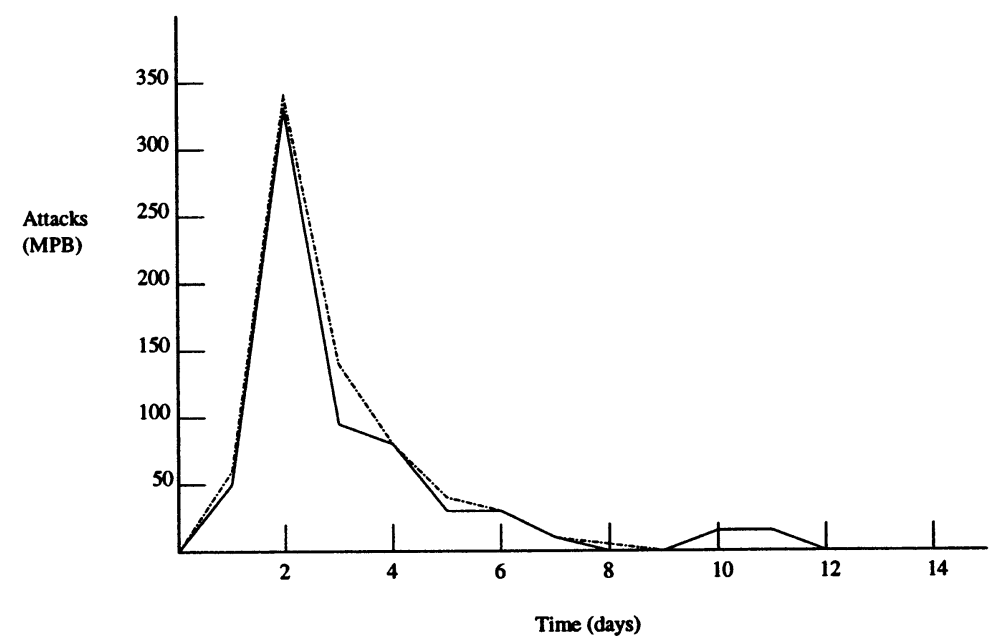

Fig. 6. Comparison of manipulated experimental data $\left(\left\{q_{n}, \ln \left(\left(A_{n} r_{0}\right) /\left(r_{1} r_{n}\right)\right)\right\}\right.$, solid line), solid line) and model predictions $\left(\left\{q_{n}\right.\right.$, $\left.\ln \left(I\left(q_{n}\right)\right)\right\}$, dashed line) of MPB attacks on an entire tree using the direct fitting procedure and the attack series for tree 1 from the 1995 SNRA data.

\author{
Calculate absolute error be- \\ tween model predictions and \\ data,$$
\text { error }=\sum_{n=1}^{\text {all days }}\left|r_{1} \frac{r(n)}{r_{0}} I\left(q(n), \gamma_{n}\right)-A_{n}\right|
$$ \\ Store current values $\{\Gamma$, error $\}$ \\ Increment $\Gamma$ \\ End Loop 3 \\ Choose smallest error measure- \\ ment with the corresponding $\Gamma$ and \\ store them with current values of \\ $\mathrm{A} 0$ and $\mathrm{V}$ : $\{\mathrm{A} 0, \mathrm{~V}, \Gamma$, minimum \\ error\} \\ Increment $\mathrm{v}$ \\ End Loop 2 \\ Increment A0

\section{End Loop 1} \\ Choose values of $\mathrm{AO}$ and $\mathrm{V}$ correspond- \\ ing to the smallest of all the 'mini- \\ mum errors'
}

Decreasing the increment sizes of $A_{0}, v$ and $\Gamma$ increases the accuracy of the final estimates, but also increases the time required to try all parameter combinations. A graphical example of this method's results can be seen in Fig. 6. We chose increments to provide one decimal place accuracy.

\section{Results and discussion}

Of the 27 trees in the 1995 SNRA plot, eight were successfully colonized by MPB. The indirect fitting method only converged for seven trees (Table 3). The weighted average for $A_{0}$ was 6.7 $\mu \mathrm{g} / \mathrm{ha}$. Averages are calculated by weighting each value's contribution according to its corresponding $r^{2}$ value. For $A_{0}$ this is:

Weighted average $=\sum_{j=1}^{\text {all }} \sum_{j=1}^{\text {trees }}\left(\frac{r_{j}^{2}}{\sum_{i=1}^{\text {all trees }} r_{i}^{2}}\left(A_{0}\right)_{j}\right)$

Estimated $A_{0}$ values range from 3.8 to 13.4 $\mu \mathrm{g} / \mathrm{ha}$. Values of $v$ show less variation, ranging from 2.5 to $7.0 \mathrm{ha}^{2} / \mu \mathrm{g} / \mathrm{fh}$, with an average of 5.4 $\mathrm{ha}^{2} / \mu \mathrm{g} / \mathrm{fh}$.

Using the direct method, estimates of $A_{0}$ and $v$ were obtained for all eight trees (Table 3). This method resulted in greater ranges in both parameter estimates, but consistently showed higher correlation coefficients. Estimates for $A_{0}$ ranged from 1.1 to $28.8 \mu \mathrm{g} / \mathrm{ha}$ and have a weighted average of 
Table 3

Estimates for $A_{0}(\mu \mathrm{g} / \mathrm{ha})$ and $v\left(\mathrm{ha}^{2} / \mu \mathrm{g} / \mathrm{fh}\right)$ obtained by applying the indirect and direct fitting methods to MPB attack series from the SNRA, 1995 plot $^{\mathrm{a}}$

1995 Parameter estimates

\begin{tabular}{|c|c|c|c|c|c|c|c|}
\hline & \multirow[b]{2}{*}{ Tree } & \multicolumn{3}{|c|}{ Indirect } & \multicolumn{3}{|c|}{ Direct } \\
\hline & & $A_{0}$ & $v$ & $r^{2}$ & $A_{0}$ & $v$ & $r^{2}$ \\
\hline \multirow[t]{8}{*}{ SNRA } & 1 & 3.8 & 7.0 & 0.95 & 5.5 & 3.8 & 0.98 \\
\hline & 2 & $* *$ & $* *$ & - & 13.2 & 0.3 & 0.79 \\
\hline & 3 & 6.9 & 5.9 & 0.89 & 28.8 & 1.3 & 0.96 \\
\hline & 4 & 4.7 & 6.0 & 0.05 & 1.1 & 9.2 & 0.96 \\
\hline & 5 & 13.4 & 2.5 & 0.45 & 15.0 & 1.1 & 0.78 \\
\hline & 6 & 6.2 & 5.1 & 0.72 & 16.8 & 1.3 & 0.96 \\
\hline & 7 & 7.1 & 4.6 & 0.98 & 8.8 & 5.7 & 0.99 \\
\hline & 8 & 5.5 & 6.2 & 0.33 & 3.8 & 4.1 & 0.93 \\
\hline \multicolumn{2}{|c|}{ Weighted average } & 6.7 & 5.4 & - & 11.5 & 3.5 & - \\
\hline
\end{tabular}

${ }^{a}$ Averages are calculated using associated $r^{2}$ values, according to Eq. (12)

** Indicates values for which the indirect fitting technique failed to converge.

$11.5 \mu \mathrm{g} / \mathrm{ha}$. The maximum and minimum differ by about 28 units, about three times the range associated with the indirect fitting method.
Ranging from 0.3 to $9.2 \mathrm{ha}^{2} / \mu \mathrm{g} / \mathrm{fh}, v$ differs by almost nine units. The weighted average is 3.5 $\mathrm{ha}^{2} / \mu \mathrm{g} / \mathrm{fh}$.

In plot 1 of the 1996 St. Charles experiments, only the focus tree, out of a total of 40 trees, was successfully colonized by MPB. That year, plot 2 had only eight of 35 trees colonized. Indirectly fitting the model to these nine trees' attack series resulted in estimates from only four (Table 4). Compared with 1995, this year's indirect estimates for $A_{0}$ show less variability while estimates for $v$ show more. Estimates for $A_{0}$ range from 2.9 to 6.2 $\mu \mathrm{g} / \mathrm{ha}$, slightly over three units. The weighted average is $3.5 \mu \mathrm{g} / \mathrm{ha}$. Estimates for $v$ range over about 13 units from 5.3 to $17.8 \mathrm{ha}^{2} / \mu \mathrm{g} / \mathrm{fh}$ with an average of $12.0 \mathrm{ha}^{2} / \mu \mathrm{g} / \mathrm{fh}$. The direct fitting technique had a higher success rate. Estimates were obtained from all nine trees' attack series. Ranging from 0.7 to $14.4 \mu \mathrm{g} / \mathrm{ha}$, estimates for $A_{0}$ have a weighted average of $3.8 \mu \mathrm{g} / \mathrm{ha}$. The parameter $v$ has an average of $10.5 \mathrm{ha}^{2} / \mu \mathrm{g} / \mathrm{fh}$ and ranges from 6.5 to $18.6 \mathrm{ha}^{2} / \mu \mathrm{g} / \mathrm{fh}$.

During the 1997 experiments, plot 1 near St. Charles had six trees out of 18 colonized. Of 16 host trees in plot 2, 11 were successfully colonized. The indirect fitting procedure converged upon parameter estimates for 16 of the seventeen

\section{Table 4}

Estimates for $A_{0}(\mu \mathrm{g} / \mathrm{ha})$ and $v\left(\mathrm{ha}^{2} / \mu \mathrm{g} / \mathrm{fh}\right)$ obtained by applying the indirect and direct fitting methods to MPB attack series from two plots in St. Charles, ID, 1996

1996 Parameter estimates

\begin{tabular}{|c|c|c|c|c|c|c|c|}
\hline & Tree & $A_{0}$ & $v$ & $r^{2}$ & $A_{0}$ & $v$ & $r^{2}$ \\
\hline St. Charles plot 1 & 1 & 3.4 & 8.8 & 0.76 & 4.2 & 18.6 & 0.98 \\
\hline \multirow{6}{*}{ Plot 2} & 1 & 2.9 & 17.8 & 0.77 & 3.5 & 8.7 & 0.86 \\
\hline & 2 & 2.5 & 13.9 & 0.94 & 1.1 & 6.5 & 0.93 \\
\hline & 5 & $* *$ & $* *$ & - & 0.7 & 8.8 & 0.97 \\
\hline & 6 & $* *$ & $* *$ & - & 0.7 & 11.8 & 0.80 \\
\hline & 7 & $* *$ & $* *$ & - & 0.7 & 12.6 & 0.62 \\
\hline & 8 & 6.2 & 5.3 & 0.58 & 5.1 & 6.7 & 0.97 \\
\hline
\end{tabular}

a Averages are calculated using associated $r^{2}$ values, according to Eq. (12).

** Indicates values for which the indirect fitting technique failed to converge. 
St. Charles trees (Table 5). Values of $A_{0}$ range from 5.0 to $11.5 \mu \mathrm{g} / \mathrm{ha}$, a range of about seven units. The weighted average for these two plots is $8.4 \mu \mathrm{g} / \mathrm{ha}$. Spanning just under 11 units from 0.6 to $11.9 \mathrm{ha}^{2} / \mu \mathrm{g} / \mathrm{fh}, v$ has an average of $3.7 \mathrm{ha}^{2} / \mu \mathrm{g} /$ fh. Applying the direct fitting technique to the same attack series, $A_{0}$ estimates range from 1.7 to $6.7 \mu \mathrm{g} / \mathrm{ha}$ and averages to $4.4 \mu \mathrm{g} / \mathrm{ha}$. Estimates for $v$ range over about 13 units from 1.7 to 14.2 $\mathrm{ha}^{2} / \mu \mathrm{g} / \mathrm{fh}$. The weighted average for $v$ is $4.7 \mathrm{ha}^{2} /$ $\mu \mathrm{g} / \mathrm{fh}$.

The same year, SNRA plot 1 had only four of 27 available hosts colonized (Table 6). Plot 2 had a high percentage of trees colonized. Unfortunately, MPB activity in this plot began before we commenced making observations and part of the temporal structure of most attack series was lost. We were able to obtain indirect estimates of four trees' parameter values. In plot 3 , of 30 hosts, only five were successfully mass attacked and colonized. Of these five, only the attack series from the focus tree resulted in indirect parameter estimates. Since the direct fitting method is based upon finding the lowest point of an error surface in parameter space and there must always be a lowest point, estimates for all 25 attack series were obtained.

The fact that the direct fitting method's correlation coefficients for plot 2 are so high suggests that this technique is not sensitive to the loss of the initial temporal structure of an attack series. To determine if these high $r^{2}$ values are an artifact of the direct fitting algorithim we fitted the model to ten randomly generated attack series of ten 'trees' in a simulated plot of 20 trees. These random attack series produced a wide range of parameter estimates using the indirect and direct fitting techniques, with the associated correlation coefficients consistently $r^{2}=0.50$ or less and only two of them above $r^{2}=0.20$.

Table 5

Estimates for $A_{0}(\mu \mathrm{g} / \mathrm{ha})$ and $v\left(\mathrm{ha}^{2} / \mu \mathrm{g} / \mathrm{fh}\right)$ obtained by applying the indirect and direct fitting methods to MPB attack series from two plots near St. Charles, ID, $1997^{\mathrm{a}}$

1997 St. Charles parameter estimates

\begin{tabular}{|c|c|c|c|c|c|c|c|}
\hline & \multirow[b]{2}{*}{ Tree } & \multicolumn{3}{|c|}{ Indirect } & \multicolumn{3}{|c|}{ Direct } \\
\hline & & $A_{0}$ & $V$ & $r^{2}$ & $A_{0}$ & $v$ & $r^{2}$ \\
\hline \multirow[t]{4}{*}{ St. Charles plot 1} & 1 & 5.8 & 5.7 & 0.95 & 3.1 & 3.7 & 0.99 \\
\hline & 2 & 5.0 & 11.9 & 0.28 & 2.1 & 2.7 & 0.98 \\
\hline & 3 & 5.0 & 2.8 & 0.62 & 1.7 & 6.3 & 0.84 \\
\hline & 6 & 5.9 & 5.1 & 0.69 & 2.7 & 5.3 & 0.99 \\
\hline \multirow[t]{7}{*}{ Plot 2} & 1 & 10.1 & 2.9 & 0.91 & 4.4 & 3.3 & 0.96 \\
\hline & 2 & 9.2 & 0.6 & 0.12 & 5.9 & 1.7 & 0.85 \\
\hline & 3 & 8.8 & 3.5 & 0.96 & 4.7 & 1.9 & 0.99 \\
\hline & 4 & 8.9 & 3.7 & 0.45 & 4.8 & 5.0 & 0.72 \\
\hline & 9 & 9.6 & 3.0 & 0.96 & 3.3 & 4.5 & 0.98 \\
\hline & 10 & 7.7 & 1.7 & 0.95 & 5.7 & 11.2 & 0.92 \\
\hline & 11 & $* *$ & $* *$ & - & 5.1 & 2.3 & 0.89 \\
\hline Weighted average & & 8.4 & 3.7 & - & 4.4 & 4.7 & - \\
\hline
\end{tabular}

a Averages are calculated using associated $r^{2}$ values, according to Eq. (12).

** Indicates values for which the indirect fitting technique failed to converge. 
Table 6

Estimates for $A_{0}(\mu \mathrm{g} / \mathrm{ha})$ and $v\left(\mathrm{ha}^{2} / \mu \mathrm{g} / \mathrm{fh}\right)$ obtained by applying the indirect and direct fitting methods to MPB attack series from three plots in the SNRA, $1997^{\mathrm{a}}$

1997 SNRA parameter estimates

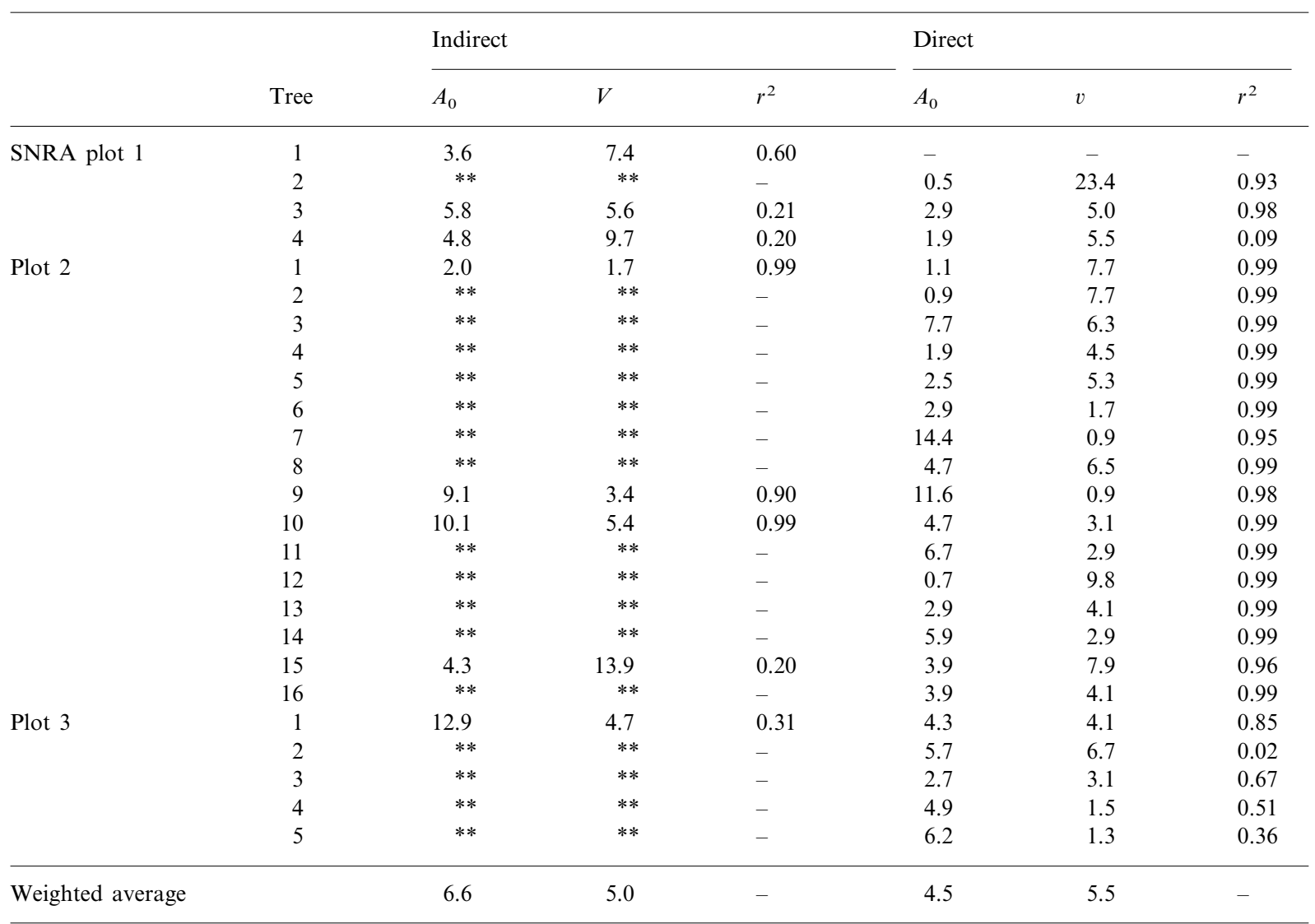

a Averages are calculated using associated $r^{2}$ values, according to Eq. (12).

** Indicates values for which the indirect fitting technique failed to converge.

Considering the three 1997 SNRA plots together, indirect estimates for $A_{0}$ range from 2.0 to $12.9 \mu \mathrm{g} / \mathrm{ha}$ with an average of $6.6 \mu \mathrm{g} / \mathrm{ha}$. Values of $v$ span about 12 units from 1.7 to 13.9 $\mathrm{ha}^{2} / \mu \mathrm{g} / \mathrm{fh}$. The weighted average is $5.0 \mathrm{ha}^{2} \mu \mathrm{g} / \mathrm{fh}$. Estimates from the direct fitting method for $A_{0}$ average to $4.5 \mu \mathrm{g} / \mathrm{ha}$ and range from 0.5 to 14.4 $\mu \mathrm{g} / \mathrm{ha}$. The average for $v$ is $5.5 \mathrm{ha}^{2} / \mu \mathrm{g} / \mathrm{fh}$ with estimates ranging from 0.9 to 23.4 , a span of about 23 units.

Considering all years' results simultaneously, indirect estimates of $A_{0}$ range from 2.0 to 13.4 $\mu \mathrm{g} / \mathrm{ha}$. Estimates of $A_{0}$ show more variation using direct parametrization, $0.5-28.8 \mu \mathrm{g} / \mathrm{ha}$. The esti- mates for $v$ show less variability, ranging from 0.6 to $17.8 \mathrm{ha}^{2} / \mu \mathrm{g} / \mathrm{fh}$ when fitted indirectly then when fitted directly; $0.3-23.4 \mathrm{ha}^{2} / \mu \mathrm{g} / \mathrm{fh}$. Table 7 is a summary of all years' weighted averages calculated using the indirect and direct techniques.

The correlation coefficient for indirect fits between predictions and data vary widely, spanning nearly the entire range of possible values. Tree 3 of the 1995 plot has an $r^{2}$ value of 0.05 , indicating a very poor fit, while tree 1 of 1997 SNRA plot 2 has an $r^{2}=0.99$, indicating a good fit. Comparing the indirect and direct fitting methods, the $r^{2}$ values obtained using the direct method are consistently higher, but at great computational cost. 
Table 7

Summary of estimates and ranges for $A_{0}(\mu \mathrm{g} / \mathrm{ha})$ and $v\left(\mathrm{ha}^{2} / \mu \mathrm{g} / \mathrm{fh}\right)$ obtained by direct and indirect fitting techniques ${ }^{\mathrm{a}}$

Summary of parameter estimates

\begin{tabular}{|c|c|c|c|c|c|c|}
\hline & $A_{0}$ & $v$ & Product & $A_{0}$ & $v$ & Product \\
\hline 1995 SNRA & 6.7 & 5.4 & 36.2 & 11.5 & 3.5 & 40.3 \\
\hline 1996 St. Charles & 3.5 & 12.0 & 42.0 & 3.8 & 10.5 & 39.9 \\
\hline 1997 St. Charles & 8.4 & 3.7 & 31.1 & 4.4 & 4.7 & 20.7 \\
\hline Combined average & 5.3 & 7.1 & 37.6 & 5.4 & 5.7 & 30.8 \\
\hline
\end{tabular}

a The combined averages represent weighted averages of all estimates, not simply an average of yearly averages. Averages are calculated using associated $r^{2}$ values, according to Eq. (12). The product of each combination of averages for $A_{0}$ and $v$ is shown for its importance in the discussion of non-dimensional combinations in Section 3.1. These products represent the multiplication of the averages and not the averages of the product of individual tree's parameter estimates.

\subsection{Parameter estimate variation}

The variation in parameter estimates was not unexpected and there are several identifiable contributing factors. Some of the basic assumptions or generalizations used to construct the model do not hold true over time or space. The approximations of five flight hours per day, although an acceptable estimate, will naturally vary depending on the location of individual plots and daily temperature regimes. Another source of variation is because we treat each tree and its associated chemotactic plume as if it was isolated from all other trees' plumes. In reality, there is certain to be some influence on the pheromone plume of one tree caused by the pheromones emitted from MPB nesting in neighbouring trees. This type of influence will vary from tree to tree depending on the juxtaposition of a tree and its neighbours. This type of interaction is beyond the scope of this paper but is being addressed in another branch of the larger MPB project.

The scale at which the local model operates is also a likely source of variation in parameter estimates, and the model fails to account for the microclimate surrounding individual trees. Not only will wind conditions on both micro- and macro-scales vary from place to place and from moment to moment, but the temperature will also vary by aspect of the tree bole and relative posi- tion in the stand. The density of a particular stand of trees will have an impact on the microclimate within the phloem of an individual tree. We fail to capture this variation when we treat all plots as having uniform tree density and use averages for the diffusion of pheromones.

The effect of different size trees is also lost in the model because all trees are assumed to be of uniform size. This assumption will have an influence on $A_{0}$ particularly. Trees of different sizes will have different carrying capacities of colonizing beetles (Cole and Amman, 1969). This carrying capacity is, at some level, related to the number of attacks that occur on a tree before beetles switch to a new tree, which in our model is described by $A_{0}$. Because the model assumes all trees to be uniform, this forces their carrying capacities to be equal as well. Consequently, when the model equations attempt to match true system dynamics, the value of $A_{0}$ is not only trying to capture the chemical components of the system, but also the effects of the size of individual trees. In this sense, $A_{0}$ is describing more of the system behaviour then was intended, thus introducing a source of variation. To alleviate this problem a term accounting for individual tree size would be necessary.

To evaluate the significance of the variation in the estimates of $A_{0}$ and $v$ we can consider the two non-dimensional combinations (Logan, 1997) in the infestation function, involving $A_{0}$ and $v$ : 


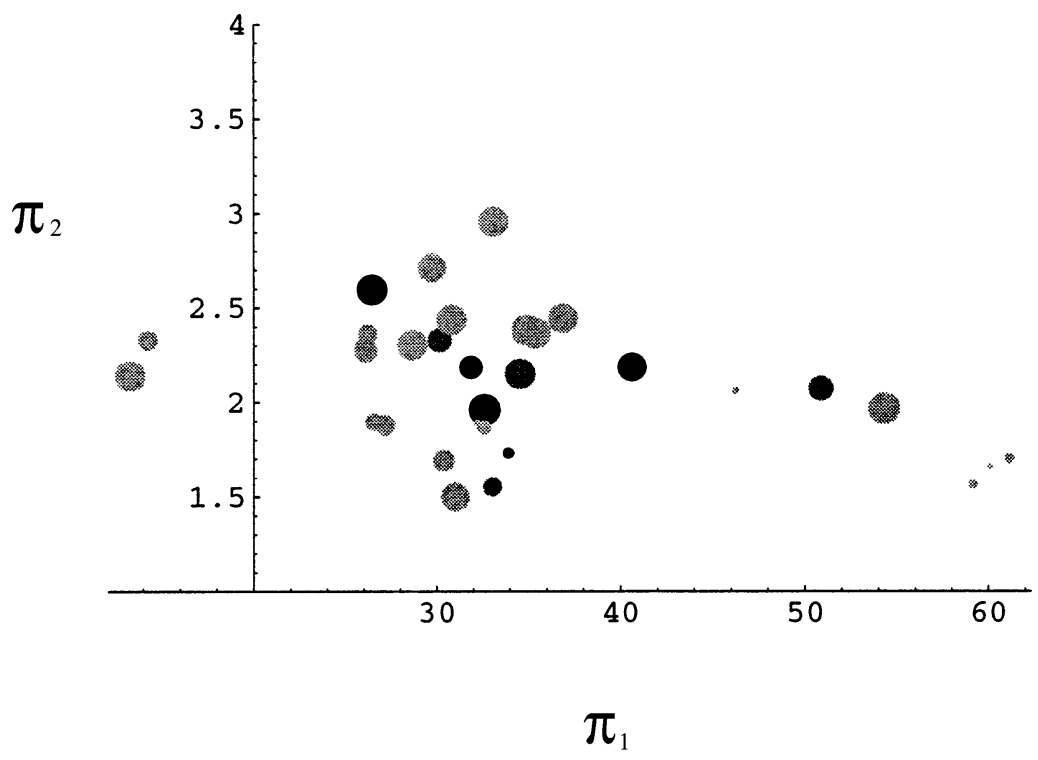

Fig. 7. Points plotted in $\pi$-space. Each point is generated using $\pi_{1}=\left(A_{0} v\right) / \mu$ and $\pi_{2}=\left[a_{1} q_{\text {final }}\right] /\left[A_{0}\left(4 b_{1}+w \delta_{1}\right)\right]$ indirectly fitted from a single tree, with the size of the point being scaled by the corresponding $r^{2}$ value. Different shades represent different years' data; dark grey points represent 1995 data, black points represent 1996 data, and light grey points represent 1997 data.

$\pi_{1}=\frac{A_{0} v}{\mu} \quad$ and $\quad \pi_{2}=\frac{a_{1} q_{\text {final }}}{A_{0}\left(4 b_{1}+w \delta_{1}\right)}$

The parameter $\pi_{1}$, can be interpreted as the relative importance of pheromone-directed and random components of MPB behaviour, while $\pi_{2}$ measures the competitive ratio of pheromone contributions by MPB and enviromental loss. With both $A_{0}$ and $v$ free to vary, the fitting procedures will actually be searching for values of $\pi_{1}$ and $\pi_{2}$ which result in the least sum-of-squares (indirect method) or the smallest absolute error (direct method). Fig. 7 shows points in $\pi$-space generated by indirect parameter estimates and MPB attack series on individual trees. Even though estimates vary widely from tree to tree, the fact that the points in $\pi$-space tend to cluster suggests that the model is capturing core behaviour but failing to capture differences due to individual trees and the microclimates surrounding them. The majority of variation is in $\pi_{1}$, which suggests that the current model needs to be improved in its representation of chernotaxis. The relatively small variation in $\pi_{2}$ indicates that the model's depiction of pheromone loss/gain is reasonably accurate. The products of averaged estimates in Table 7 represent the value of $\pi_{1}$ (since $\mu=1$ ). They are clustered at the same position along the $\pi_{1}$-axis as the individual estimates in Fig. 7.

\subsection{Comparison of fitting techniques}

When comparing the indirect and direct fitting methods it is important to recall the intended purpose of each. The indirect method can be quickly applied to many time series, sacrificing some degree of reliability. The direct method is more reliable in its estimates and may be used to determine the accuracy and overall effectiveness of the indirect method. This method, however, is much slower and more difficult to employ than the indirect method.

We use the indirect method primarily because it quickly and easily generates parameter estimates for many data sets. A weakness of this method is the many assumptions and simplifications required, which make estimated parameter values less reliable. Another problem is that the type of non-linear fitting technique used reveals nothing about the topology of the error surface upon which it is searching. It is therefore difficult to 
know whether estimates represent global or local minima. Additionally, separate calculations are required to determine the sensitivity of the model to particular parameters.

To determine the severity of the problems of the indirect method, we estimate parameter values by directly solving the model equations for systematic combinations of $A_{0}$ and $v$. This requires fewer assumptions and provides error measurements for incremental values of parameters within a specified region, generating a picture of the error surface in parameter space. Fig. 8 depicts the error surface for tree 7 from the 1995 SNRA data. This plot reveals a trend seen in all analyzed trees' error surfaces: $A_{0} v=$ constant $\sim \pi_{1}$.

The direct method almost always produced a better fit between model predictions and observed data. The correlation coefficients are very high, often 0.99. For the 1996 and 1997 trees the weighted averages from the direct method are comparable to estimates obtained from the indi- rect method (Table 7). This would suggest that despite its weaknesses, the indirect method does an acceptable job of estimating parameter values. The 1995 direct estimate of $A_{0}$ agrees less well with other estimates of $A_{0}$. It is considerably higher than expected. Mathematically, this is due to the estimated value for tree $3, A_{0}=28.8$, which is more than twice the value of the next largest estimate. Removing this tree from the 1995 average gives estimates of $A_{0}=8.9 \mu \mathrm{g} / \mathrm{ha}$ and $v=3.8$ $\mathrm{ha}^{2} / \mu \mathrm{g} / \mathrm{fh}$, which agree more closely with other years' estimates. Also, the fact that the two largest averages for $v$ result when the indirect and direct fitting methods are applied the 1996 data suggest that there may be an unmodelled biological phenomenon driving the larger estimate. Overall, the two methods appear to agree quite well in their estimates of the two parameters.

Parameter estimates for an individual tree obtained using the direct and indirect methods differ to a greater or lesser degree, but from the error

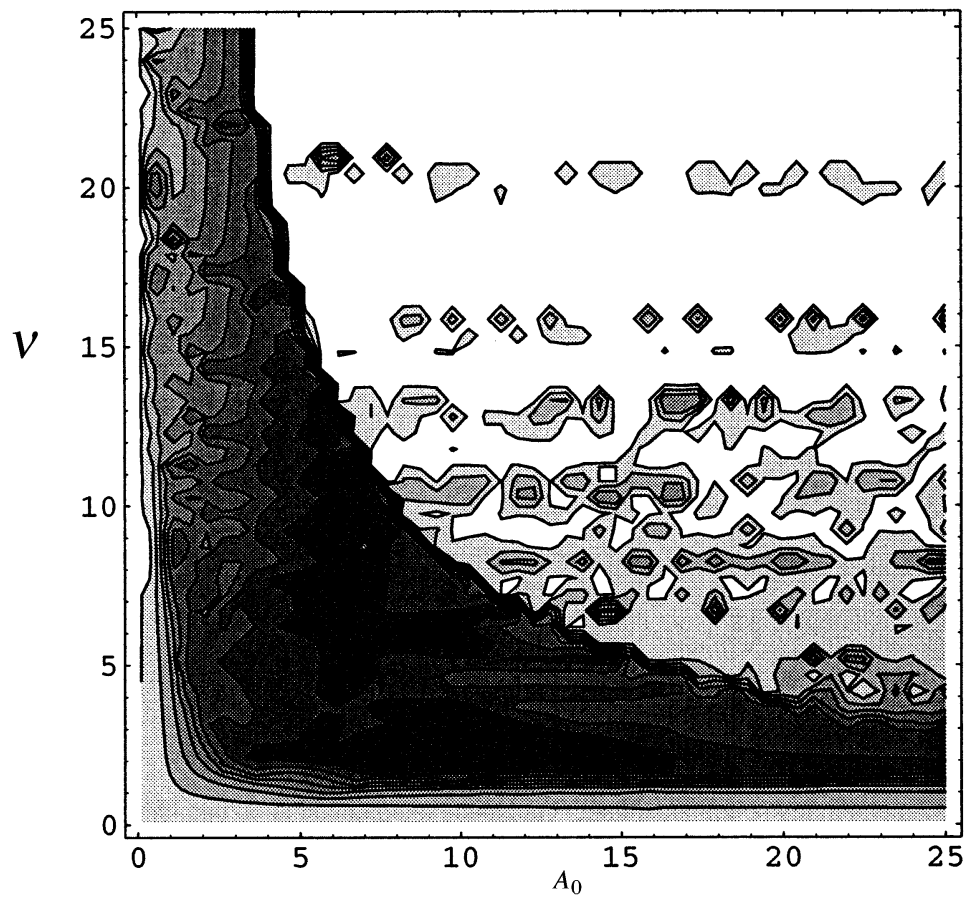

Fig. 8. Error surface contours showing the measured error between model predictions and observational data for different values of $A_{0}$ and $v$ using data from tree 7 of the 1995 SNRA plot. Darker areas represent regions of smaller error. Notice the sharp decrease in error along a curve $\pi_{1}=\left(A_{0} v\right) / \mu=$ constant. 
surfaces generated by the direct method, we see that values obtained from the indirect method lie at local minima of the surface. In some cases the local and global minima, even though they have different $A_{0}$ and $v$ values, have nearly the same associated error.

\subsection{Validation}

The best way to validate the parameterized model would be to integrate the PDE Eqs. (1)-(5) and compare with spatial data on the forest scale. This requires a complete demographic survey of hosts on a forest scale, and subsequent resurvey after flight season, which could then in principle be compared with model output. Unfortunately there are simply not resources available for such an effort. We are pursuing alternative means of validation. One approach is to investigate the coupling of two attack series at different spatial locations to determine if the parametrized model predicts how one attack series is conditioned on a previous attack series. This work is complicated in its own right and will be published separately.

In the current paper we use independent samples of final attack density at season end to test the predictivity of the parametrized 'local' model. To approximate the final number of nesting beetles we counted attacks within $0.1225 \mathrm{~m}^{2}$ at various heights on both the north and south aspects of colonized trees in both the St. Charles plots from 1997. We determined the density of attacks from the counted sections and multiplied by the total estimated area of infestation. Observed maximum attack heights ranged from 5.95 to $15.4 \mathrm{~m}$.

To produce model predictions, the values $A_{0}=$ $5.4 \mu \mathrm{g} / \mathrm{ha}$ and $v=5.7 \mathrm{ha}^{2} / \mu \mathrm{g} / \mathrm{fh}$ (Table 7) are used. To determine the shape and magnitude of $\gamma$ the procedure of the direct fitting technique was employed. Thus, $\gamma$ varied from plot to plot. Individual trees' resin reservoirs, $r_{0}$, and rates at which resin is replenished, $r_{2}$, vary with tree size, so we approximate these parameters for each tree according to the relationships developed in Powell et al. (1999). Since resin is carried in the xylem layer of a tree the constitutive resin reservoir, $r_{0}$, is considered to be proportional to the ratio of xylem volume and the surface area of the tree. This ratio results in:

$r_{0} \sim W\left(1-\frac{\mathrm{LR}}{\mathrm{DBH}}\right) \mathrm{LR}$

where $W$ is the limiting growth factor, LR is the approximate depth of living wood $(10.35 \mathrm{~cm})$ and $\mathrm{DBH}$ is the diameter at breast height of the tree. We choose to normalize $r_{0}$ so that a $25.4 \mathrm{~cm} \mathrm{DBH}$ tree under no stress has $r_{0}=1$.

The rate of resin replenishment depends on the amount of 'free energy' the tree has which can be directed towards resin production. In volumetric units this should be proportional to the ratio of new wood volume to the living wood volume. Thus, Powell et al. (1999) define a unitless tree 'vigour', $V$, by:

$V \stackrel{\text { def }}{=} \frac{\mathrm{BAI}}{\pi(\mathrm{DBH}-\mathrm{LR}) \mathrm{LR}}$

where BAI is a species-specific constant describing the basal area a tree can grow in one year. The resin replenishment rate of a tree will be proportional to the tree's vigour. Again, we normalize the $r_{2}$ so that a $25.4 \mathrm{~cm} \mathrm{DBH}$ lodgepole under no stress will have $r_{2} / w=0.1$ per $\mathrm{fh} / R_{0}$.

Using the observed $\mathrm{DBH}$ from individual trees we calculated values for $r_{0}$ and $r_{2}$ with which to predict the final number of beetles nesting in a tree. From 1997 St. Charles plots 1 and 2, the observed final numbers of nesting beetles are shown in Table 8. Overall, model predictions appear to adequately match observed data.

\subsection{Biological interpretation of parameters}

Our best estimate of $v$ is $5.7 \mathrm{ha}^{2} / \mu \mathrm{g} / \mathrm{fh}$. This parameter describes the strength of chemotactic attraction to the pheromones of nesting beetles. Its ecological significance is perhaps most easily understood through the role it plays in $\pi_{1}$. The numerator of this combination, $A_{0} v$, describes MPB movement as directed by chemical cues. The denominator, $\mu$, describes undirected beetle movement. The dimensionless combination, $\pi_{1}$, may therefore be thought of as the ratio of the influence of chemical cues to the influence of randomness in beetle movement. A value of $\pi_{1}=30.8$ 
Table 8

Observed and predicted numbers of MPB nesting in colonized trees after the summer flight season has been completed $^{\text {a }}$

1997 St. Charles final attacks

\begin{tabular}{lrrl}
\hline & Tree & Observed & Predicted \\
\hline Plot 1 & 1 & 1496 & 958 \\
& 2 & 923 & 911 \\
& 3 & 631 & 949 \\
& 4 & 910 & 961 \\
Plot 2 & 5 & 1391 & 907 \\
& 6 & 567 & 933 \\
& 1 & 1300 & 987 \\
& 2 & 1138 & 987 \\
& 3 & 1076 & 943 \\
& 4 & 722 & 850 \\
& 5 & 863 & 944 \\
6 & 704 & 931 \\
& 7 & 728 & 964 \\
& 8 & 791 & 887 \\
& 9 & 693 & 944 \\
& 10 & 987 & 922 \\
\hline 11 & 760 & \\
\hline
\end{tabular}

a These trees are from the 1997 St. Charles plots. Predictions are made using the weighted average parameter estimate and not specific estimates for each tree.

means that beetle movement is directed about 31 times more strongly by pheromones than by random redistribution, which seems completely reasonable for an organism which relies so heavily on spatial aggregation.

The parameter $A_{0}$ is more difficult to interpret ecologically. For simplicity our model treats the MPB pheromone communication system as if it employs only one pheromone, which in reality is a surrogate for a suite of pheromones. We assume this single pheromone to be responsible for both the aggregation and anti-aggregation of MPB attacks on a single tree dependent upon its local concentration. In these terms, the value $A_{0}=5.4 \mu \mathrm{g} / \mathrm{ha}$ is easily interpreted. Below this concentration MPB attacks are focused upon the host tree; above this concentration MPB are discouraged from attacking. The true pheromone communication system involves multiple chemicals, each existing at its own concentration and exerting its own influence on flying MPB. Our estimate of $A_{0}$ is not directly interpretable as the concentration of any one pheromone; instead, it represents the point in an attack series at which the cumulative pheromone effects switch from being focusing to de-focusing. Using the relationship between the global and local models, from Eq. (5) (Powell and Rose, 1997), pheromone concentrations at a single location, $A$, can be written as a function of the number of nesting beetles, $q$, nesting in a tree in the following way:

$$
A=\frac{2 a_{1} q}{4 b_{1}+w \delta_{1}}
$$

If $A_{0}$ is the point at which pheromone effects switch from being aggragative to anti-aggragative, by assigning it a value we can determine the number of beetles nesting in a tree when this switching occurs. Setting $A \stackrel{\text { set }}{=} A_{0}=5.4$ gives:

$q_{\text {switch }} \doteq \frac{5.4\left(4 b_{1}+w \delta_{1}\right)}{2 a_{1}} \approx 420 \mathrm{MPB}$

This suggests that switching occurs at a little less than half of the 'carrying capacity' of a tree, which the model consistently predicts to be around 930 MPB. These results corroborate with field data (Bentz et al., 1996). At this point, attacks still occur on the initial tree, but at a much slower rate.

\section{Conclusion}

In this paper we have used localization and two independent fitting procedures to estimate behavioural parameters for a spatially extended, partial differential model for MPB dispersal and subsequent mass-attack on individual host pines. The major challenge we have overcome is using point measurements (number of attacks on individual trees) to determine parameters $\left(A_{0}, v\right)$ which can only be understood in terms of their impact on spatially extended and averaged behaviours of densities of organisms. This was accomplished, and local validation measures used, to give some degree of confidence in our parameter predictions. In fact, considering the plethora of non-behavioural parameters which 
had to be estimated, the localized model did astonishingly well.

The sources of variation in parameter estimation using the local model are not necessarily fatal problems. In the end we wish to obtain estimates to be used in the global model to describe system behaviour in an entire forest, not to describe system behaviour at a single location. The use of averages (for wind speed or stand density, for example) make the estimated parameter values generally applicable and useful in the global model.

Of course, it would have been much more direct to have spatially extended measurements to parameterize the model, like those used by Turchin and Simmons (1998) and Cronin et al. (1997) for southern pine beetle. We attempted these measurements for MPB and failed; we were unable to collect any reliable information on population density gradients in any of the 3 years of field observation. This underscores the importance of alternate strategies for parameterization.

There is a clear need for more work on parameterizing partial differential models of ecological systems. While there has been a wealth of models constructed, often with suggestive or illuminating behaviour, very little attention has been paid to problems associated with determining parameter values for these models. Without parameterization all of the suggestive modelling amounts only to consciousness-raising, and cannot assist with practical questions in ecology.

It is those sorts of questions this work now frees us to address. The 'global' partial differential model for MPB dispersal can be used to investigate ideas relating to management and risk in this important forest ecosystem. Coupled with realistic models of forest and insect phenology the global dispersal model can be used to help understand factors contributing to population outbreaks. Finally, given the current nature of forest demographics, parameterized and validated spatial models will be required to envision and role-play scenarios for managing western forests back to a self-maintaining system in which MPB outbreaks play a healthy and self-limiting part.

\section{Acknowledgements}

Dr Powell and Mr Biesinger were supported by NSF grant DMS-9505327, an REU supplement and the USDA Forest Service Mountain Pine Beetle Project. Drs Bentz, Logan and Powell were supported by NSF grant DMS-9505327 and the Utah Mineral Lease Replacement Program.

\section{Appendix A. Estimating 'local' parameter values}

\section{A.1. Chemical diffusion parameters}

The model function (Powell et al., 1996) for chemotactic flux of the MPB population is:

$\Phi=v P \frac{A_{0}-A}{A_{0}+A / A_{3}} \nabla A$

This flux function has the effect of attracting beetles for small $A$ and dispersing beetles for large $A$, parametrized by $A_{3}$ for $A>A_{0}$. We have chosen $A_{3}=1$ so that when $A$ gets large the flux is approximately:

$\Phi=-v A_{3} P \nabla A$

and as $A$ gets small the flux is approximately:

$\Phi=v P \nabla A$

Since $v$ is the velocity of response of the population to a given pheromone gradient, $A_{3}$ gives equal peak response velocities for either aggregation or anti-aggregation.

To estimate the rate of pheromone production, $a_{1}$, we refer to Borden et al. (1986). They estimate the production rate of trans-verbenol by a female MPB at about $20 \mathrm{ng} / \mathrm{fh}$, giving:

$a_{1}=2 \mu \mathrm{g} / \mathrm{fh} / \mathrm{HMPB}$

The parameters $b_{1}$ and $\delta_{1}$, the rate of pheromone diffusion and the loss rate of pheromone, respectively, can be estimated simultaneously. The self-modifying spatial behaviour of the system is mediated by the pheromone plume produced by nesting MPB. One model for the diffusion of the pheromone plume produced by $q$ nesting MPB at the origin would be: 
$A_{t}-u A_{x}=b_{1} A_{y y}+b_{2} A_{x x}-\delta_{1} A+a_{1} q \delta(x, y)$

where $u$ is the average wind speed, which we will assume is directed along the $x$-axis in the positive direction. We also assume, based on observations of smoke plumes, that the diffusion in the direction of the wind itself is small $\left(b_{2} \approx 0\right)$. Taking $a_{1}$ to be $2 \mu \mathrm{g} / \mathrm{fh} / \mathrm{HMPB}$ to determine the remaining parameters, $b_{1}$ and $\delta_{1}$ we rely on dimensional arguments. As in Tennekes and Lumley (1975), let $\xi$ be the average separation between hosts $(\sim 3$ $\mathrm{m})$ in an open-canopy stand. We estimate the scale of losses to satisfy:

$\xi^{2} \simeq \frac{b_{1}}{\delta_{1}}$

so that characteristic losses occur on a tree-to-tree scale in open-stand conditions. The rate of chemical mixing due to turbulence we relate to the adation generated by solution via method of steepest descents. Solving these two expressions for $b_{1}$ and $\delta_{1}$ gives:

$b_{1}=\frac{u \xi}{2} \quad$ and $\quad \delta_{1}=\frac{u}{2 \xi}$

As the canopy of a stand becomes more closed the air below the canopy becomes more isolated from the air above. While for a particular choice of $u$ this should not change the rate of horizontal diffusion, it will influence the rate of loss through the canopy. We therefore augment the descriptions of $b_{1}$ and $\delta_{1}$ with a scaling factor, $\sigma$, reflecting the degree of closure of the canopy $(\sigma=1$ indicates open-stand conditions, $\sigma=0$ indicates a solid canopy). Choosing an average wind speed of $u=0.6 \mathrm{~m} / \mathrm{s}$ and an average spatial scale of loss of $\xi=3 \mathrm{~m}$ gives $b_{1}=0.324 / \sigma$ ha $/ \mathrm{fh}$ and $\delta_{1}=360 \sigma$ per fh. We use an average value of $\sigma=0.5$. From an informal sensitivity analysis, variations in $\sigma$ have only a small effect on parameter estimates.

\section{A.2. Resin response parameters}

The parameter $r_{0}$ describes the constitutive resin reservoir of a lodgepole of $25.4 \mathrm{~cm}$ diameter at breast height under no environmental stress, and can be related to the volume of resin in a tree. We scale all measures of resin capacity to the health of a vigourous, unstressed tree, setting $r_{0}=1$ for a $25.4 \mathrm{~cm}$ lodgepole, which is approximately the average size in our plots. To simplify unit analysis this can be denoted by units of [ $w R_{0}$ ], where $R_{0}$, a global parameter, describes volume of resin per area, so that:

$r_{0}=w R_{0}=1$

The size of a tree being attacked is described by $w$. It is important to note that this is not the basal area or crown area of a tree, but a description of the area in which a tree is the dominant feature. A reasonable estimate of this is $3 \mathrm{~m}^{2}$ cross-sectional area.

To estimate the mortality rate of MPB due to host defenses, $\beta$, the rate of resin replenishment and outflow through attack holes, $r_{2}$ and $r_{3}$, respectively, and the rate of resin crystallization, $r_{4}$, we write and solve a system of equations involving these four parameters.

The first system equation is written assuming that a general attack rate of 5 HMPB over a 5-day period is just barely sufficient for overwhelming a healthy tree's defenses (Wood, 1972; Berryman, 1978). Estimating there to be five flight hours (fh) in a day, this translates into an infestation rate of $0.2 \mathrm{HMPB}$ per flight hour. Using this in Eq. (6) in place of the infestation term should correspond to $\dot{q}=0$ giving:

$0 \frac{\mathrm{HMPB}}{\mathrm{fh}}=0.2 \frac{\mathrm{HMPB}}{\mathrm{fh}}-\beta \frac{r_{3}}{w} q r$

At this point the tree should be able to replenish its resin reserves at the same rate as they are being depleted by attacking beetles, thus no net change should be seen. From the steady state of Eq. (8) we have $r=r_{0}-h r_{3} / r_{2}$. Assuming the number of attack holes to be approximately equal to the number of attacking female beetles and replacing $r$ in Eq. (A3) results in:

$$
0.2 \frac{\mathrm{HMPB}}{\mathrm{fh}}=\beta \frac{r_{3}}{w} r_{0} q\left(1-\frac{r_{3}}{r_{0} r_{2}} q\right)
$$

In this form, the resin capacity of a tree is a function of numbers of nesting beetles,

$f(q)=q\left(\frac{r_{3}}{r_{0} r_{2}} q\right)$. 
This quadratic has zeros at $q=0$ and $q=r_{0} r_{2} / r_{3}$, and has the form of a parabola with its maximum midway between the zeros, meaning that the maximum response of the tree will be when $q=r_{0} r_{2} /$ $2 r_{3}$. Using this value in Eq. (A4) we have:

$0.2 \frac{\mathrm{HMPB}}{\mathrm{fh}}=\frac{\beta r_{2}}{4 w}$

where $r_{0}$ has been replaced with its value of 1 . Unit analysis reveals the units of $\beta$ to be per ha $/ R_{0}$.

Since global model variables describe densities rather than numbers at a point, the parameters $r_{2}$ and $r_{3}$ must also describe rates involving densities. To use them in the local model they must be converted to rates involving numbers. This can be accomplished by dividing by $w$, the characteristic size of a tree. To avoid introducing new parameters we use the ratios $r_{2} / w$ and $r_{3} / w$. Assuming a tree to be under no stress, $r_{2}$ should be approximately equal to $r_{3} r_{4}$, meaning that the rate at which a tree can replenish its resin reserves should be about equal to the rate at which it flows out and crystallizes. That is:

$r_{2}=r_{3} r_{4}$

The third system equation is obtained by considering Eq. (8). This has steady states at $r=0$ and $r=r_{0}-h r_{3} / r_{2}$. The solution of interest is:

$r=r_{0}-\frac{r_{3}}{r_{2}} h$

Observing that there should be a value for $h$ which is sufficient to deplete the constitutive resin capacity of the tree, we estimate this value to be about 2 HMPB (Cole, 1962; Reid, 1963; Klein et al., 1978; Raffa and Berryman, 1979). So, when $r=0, h=2$, with $r_{0}=1$, we have:

$\frac{r_{3}}{r_{2}}=0.5$ ha $R_{0}$

For the final equation we estimate that it requires 1-2 days (5-10 fh) for resin to crystallize. Recalling Eq. (7) and taking there to be no continued infestation of an initally attacked, healthy tree $\left(r=r_{0}=1\right)$, we have:

$$
\dot{h}=-\frac{r_{3}}{w} r_{4} h
$$

The solution to this differential equation is ( $h$ is dependent upon time):

$h=h_{0} \exp \left(-\frac{r_{3}}{w} r_{4} \Delta t\right)$

Assuming that after two days the number of holes left unfilled by resin should be nearly zero, we can say that this should be approximately equal to $h=h_{0} \exp (-1)$. Comparing these two equations gives the relationship:

$10 \frac{r_{3}}{w} r_{4}=1$

which results in an estimate of:

$\frac{r_{3}}{\omega} r_{4}=0.1 \mathrm{ha} / \mathrm{fh} / R_{0}$

This equation completes our system involving $\beta, r_{2}, r_{3}$, and $r_{4}$ :

$\frac{\beta r_{2}}{4 w}=0.2$

$r_{2}=r_{3} r_{4}$

$\frac{r_{3}}{r_{2}}=0.5$

$\frac{r_{3}}{\omega} r_{4}=0.1$

Solving this system we estimate:

$\beta=8$ per ha $/ R_{0}$

$\frac{r_{2}}{w}=0.1$ per ha $/ R_{0}$

$\frac{r_{3}}{w}=0.05$ per fh

$r_{4}=2$ per ha $/ R_{0}$

\section{A.3. $M P B$ response parameters}

To estimate the diffusitivity of flying beetles due to random redistribution we refer to experiments of Turchin and Thoeny (1993). They estimate a parameter for the southern pine beetle, $B$, which is related to the ratio of diffusion rate $(\mu)$ and loss 
rate of the population $\left(r_{1}+\omega_{1}\right)$ in the following way:

$$
B \equiv \sqrt{\frac{D}{\delta}}=\sqrt{\frac{\mu}{r_{1}+\omega_{1}}}
$$

where $D$ is their diffusion rate, and $\delta$ is their rate at which beetles are lost from the dispersing population.

Their estimates of $B$ range from 0.19 to 4.8 per ha. Taking $B=2.4$ per ha and our values of $\omega_{1}=0.014$ per fh and $r_{1}=0.16$ per fh we can solve for $\mu$ :

$\mu=1.0 \mathrm{ha} / \mathrm{fh}$

To estimate the rate at which flying MPB land due to randomness only and not in response to chemical cues, we assume that $15 \%$ of the flying beetles will randomly land and attack trees per flight hour. At any time, $t$, the population of flying beetles can be written:

$$
P_{t}=-r_{1} P
$$

The solution to this is:

$$
P=P_{0} e^{-r_{1} t}
$$

At time $t=1$ fh the population should only have decreased by $15 \%$, leaving $85 \%$ of the original population. Substituting these in the solution gives:

$$
0.85 P_{0}=P_{0} e^{-r_{1}}
$$

Solving for $r_{1}$ gives:

$$
r_{1}=-\ln (0.85) \text { per fh }=0.16 \text { per fh }
$$

We estimate the maximum distance away from a tree a flying beetle can be and still be visually attracted to it as $r_{e}=2 \mathrm{~m}$. This acts as a conversion factor for transforming the density of flying MPB into the number of MPB attacking a tree.

\section{References}

Amman, G.D., Cole, W.E., 1983. Mountain pine beetle dynamics in lodgepole pine forests. part II: population dynamics. USDA Forest Service General Technical Report INT-145, 59.

Bentz, B.J., 1995. Rocky Mountain Research Station, Logan Forestry Sciences Lab, Logan, UT. Unpublished data.
Bentz, B.J., Powell, J.A., Logan, J.A., 1996. Localized spatial and temporal attack dynamics of the mountain pine beetle (Dendroctonus ponderosae) in lodgepole pine. USDA Forest Service Research Paper INT-RP-494, December.

Berryman, A.A., 1976. Theoretical explanations of mountain pine beetle dynamics in lodgepole pine forests. Environ. Entomol. 5 (6), 1225-1233.

Berryman, A.A., 1978. Towards a theory of insect epidemiology. Res. Popul. Ecol. 19, 181-196.

Berryman, A.A., 1982. Biological control, thresholds and pest outbreaks. Environ. Entomol. 11, 544-549.

Berryman, A.A., Raffa, K.F., Millstein, J.A., Stenseth, N.C., 1989. Interaction dynamics of bark beetle aggregation and conifer defense rates. OIKOS 56, 256-263.

Berryman, A.A., Stenseth, N.C., Wollkind, D.J., 1984. Metastability of forest ecosystems infested by bark beetles. Res. Popul. Ecol. 26 (1), 13-29.

Borden, J.H., Hunt, D.W.A., Miller, D.R., Slessor, K.N., 1986. Orientation in forest Coleoptera: an uncertain outcome of responses by individual beetles to variable stimuli. In: Payne, T.L., Birch, M.C., Kennedy, C.E.J. (Eds.), Mechanisms in Insect Olfaction. Oxford University Press, UK, pp. 97-109.

Borden, J.H., Ryker, L.C., Chong, L.J., Pierce, H.D., Johnston, B.D., Oehlschlager, A.C., 1987. Response of the mountain pine beetle, Dendroctonus ponderosae, to five semiochemicals in British Columbia lodgepole pine forests. Can. J. For. Res. 17, 118-128.

Burnell, D.G., 1977. A dispersal-aggregation model for mountain pine beetle in lodgepole pine stands. Res. Popul. Ecol. 19, 99-106.

Cole, W.E., 1962. The effects of intraspecific competition within mountain pine beetle broods under laboratory conditions. Research Note INT-97, USDA Forest Service.

Cole, W.E., Amman, G.D., 1969. Mountain pine beetle infestations in relation to lodgepole pine diameters. Research Note INT-95, USDA Forest Service.

Conn, J.E., Borden, J.H., Scott, B.E., Friskie, L.M., Pierce, H.D., Oehlschlager, A.C., 1983. Semiochemicals for the mountain pine beetle, Dendroctonus ponderosae, in British Columbia: field trapping studies. Can. J. For. Res. 13, $320-324$.

Cronin, J.T., Turchin, P., Hayes, J.L., Steiner, C.A., 1997. Intra- and inter-infestation dispersal by the southern pine beetle (submitted) Environmental Entomology 1999 June 28 (3), 496-504.

Ellner, S., Turchin, P., 1995. Chaos in a noisy world: new methods and evidence from time-series analysis. Am. Nat. $145,343-375$.

Gause, G.F., 1934. The Struggle for Existence. Wiliams and Wilkins, Baltimore.

Geiszler, D.R., Gallucci, V.F., Gara, R.I., 1980. Modeling the dynamics of mountain pine beetle aggregation in a lodgepole pine stand. Oecologia 46, 244-253.

Geiszler, D.R., Gara, R.I., 1978. Mountain pine beetle attack dynamics in lodgepole pine. In: Berryman, A.A., Amman, G.D., Stark, R.W. (Eds.), Theory and Practice of Moun- 
tain Pine beetle Management in Lodgepole Pine Forests: Symposium Procedures. Washington State University. Pullman, Washington.

Haefner, J.W., 1996. Modelling Biological Systems. Chapman and Hall, New York, NY.

Harrison, G.W., 1995. Comparing predator-prey models to Luckinbill's experiment with Didinium and Paramecium. Ecology 76, 357-374.

Holmes, E.E., Lewis, M.A., Banks, J.E., Veit, R.R., 1994. Partial differential equations in ecology: spatial interactions and population dynamics. Ecology 75, 17-29.

Hughes, P.R., 1973. Dendroctonus: production of pheromones and related compounds in response to host monoterpenes. Zeitschrift feur Angewandite Entomologie 73, 294-312.

Hunt, D.W.A., Borden, J.H., Lindgren, B.S., Gries, G., 1989. The role of autoxidation of alpha-pinene in the production of pheromones of Dendroctonus ponderosae. Can. J. For. Res. 19, 1275-1282.

Klein, W.H., Parker, K.L., Jensen, C.E., 1978. Attack, emergence and stand depletion trends of the mountain pine beetle in a lodgepole pine stand during an outbreak. Environ. Entomol. 7 (5), 732-737.

Libbey, L.M., Ryker, L.C., Yandell, K.L., 1985. Laboratory and field studies of volatiles released by Dendroctonus ponderosae. Zeitschrift feur Angewandite Entomologie 100, $381-392$.

Logan, J.A., White, P., Bentz, B.J., Powell, J., 1998. Model analysis of spatial patterns in mountain pine beetle outbreaks. Theor. Popul. Biol. 53, 236-255.

Logan, J.D., 1997. Applied Mathematics, second ed. John Wiley and Sons, Inc., New York, NY.

Martin, E. (Ed.), 1996. Mathematica 3.0: Standard Add-on Packages. Copublishers: Wolfram Media and Cambridge University Press.

Mattson, W.J., Addy, N.D., 1975. Phytophagous insects as regulators of forest primary production. Science 190, 515522.

McCambridge, W.F., 1967. Nature of induced attacks by the black hills beetle, Dendroctonus ponderosae (Coleoptera: Scolytidae). Annu. Meet. Entomol. Soc. Am. 60, 920-928.

Moeck, H.A., Simmons, C.S., 1991. Primary attraction of mountain pine beetle to bolts of lodgepole pine. Can. Entomol. 123, 299-304.

Murray, J.D., 1989. Mathematical Biology. Springer-Verlag, Berlin.

Nebeker, T.E., Hodges, J.D., Blanche, C.A., 1993. Host responses to bark beetle pathogen colonization. In: Schowalter, T.D., Filip, G.M. (Eds.), Beetle-Pathogen Interactions in Conifer Forests. Academic Press, pp. 157-169.

Pitman, G.B., 1971. Trans-verbenol and alpha-pinene: their utility in manipulation of the mountain pine beetle. J. Econ. Entomol. 64, 426-430.

Pitman, G.B., Vite, J.P., Kinzer, G.W., Fentiman, A.F., 1968. Bark beetle attractants: trans-verbenol isolated from Dendroctonus. Nature 218, 168-169.

Polymenopoulos, A.D., Long, G., 1990. Estimation and evaluation methods for population growth models with spatial diffusion: dynamics of mountain pine beetle. Ecol. Model. 51, 97-121.

Powell, J.A., Logan, J.A., Bentz, B.J., 1996. Local projections for a global model of mountain pine beetle attacks. J. Theor. Biol. 179, 243-260.

Powell, J.A., Rose, J.D., 1997. Local consequences of a global model for mountain pine beetle mass attack. Dyn. Stab. Syst. 1, 23-24.

Powell, J.A., Tams, J., Bentz, B.J., Logan, J.A., 1997a. Theoretical analysis of 'switching' in a localized model for mountain pine beetle mass attack. J. Theor. Biol. 194, 49-63.

Powell, J., White, P., McMillen, T., Biesinger, Z., Kennedy, B., Tams, J., Bentz, B., Logan, J., Roberts, D., 1997 b. State and implementation of the mountain pine beetle dispersal model. Technical report, Department of Mathematics and Statistics, Utah State University, Logan, Utah 94327-3900.

Powell, J., McMillen, T., White, P., 1998. Connecting a chemotactic model for mass attack to a rapid integro-difference emulation strategy. SIAM J. Appl. Math. 59, 547572 .

Powell, J.A., Kennedy, B., White, P., Bentz, B., Logan, J., Roberts, D., 1999. Mathematical elements of attack risk analysis for mountain pine beetle. J. Theor. Biol. (in press).

Preisler, H.K, Haiganoush, K., 1993. Colonization patterns of the mountain pine beetle in thinned and unthinned lodgepole pine stands. For. Sci. 39, 528-545.

Raffa, K.F., Berryman, A.A., 1983. The role of host plant resistance in the colonization behavior and ecology of bark beetles (Coleoptera: Scolytidae). Ecol. Monogr. 53, 27-49.

Raffa, K.F., Berryman, A.A., 1986. A mechanistic computer model of mountain pine beetle populations interacting with lodgepole pine stands and its implications for forest managers. For. Sci. 32 (3), 789-805.

Raffa, K.F., Berryman, A.A., 1979. Flight responses and host selection by bark beetles. In: Berryman, A.A., Safranyik, L. (Eds.), Dispersal of Forest Insects: Evaluation, Theory and Management Implications, Washington State University. Pullman, Washington, WA, pp. 213-233.

Raffa, K.F., Phillips, T.W., Salom, S.M., 1993. Strategies and mechanisms of host colonization by bark beetles. In: Schowalter, T.D., Filip, G.M. (Eds.), Beetle-Pathogen Interactions in Conifer Forests. Academic Press, NY, pp. 103-120.

Reid, R.W., 1963. Biology of the mountain pine beetle, Dendroctonus monticolae, in the East Kootenay region of British Colombia III. Interaction between the beetle and its host, with emphasis on brood mortality and survival. Can. Entomol. 95, 225-238.

Reid, R.W., Whitney, H.S., Watson, J.A., 1967. Reactions of lodgepole pine to attack by Dendroctonus ponderosae Hopkins and blue stain fungi. Can. J. Bot. 45, 1115-1116.

Renwick, J.A.A., Vite, J.P., 1970. Systems of chemical communication in Dendroctonus. Cont. Boyce Thompson Inst. 23, 355-360. 
Roberts, D.W., 1987. A dynamical systems perspective on vegetation theory. Vegetation 69, 27-33.

Roe, A.L., Amman, G.D., 1970. The mountain pine beetle in lodgepole pine forests. USDA Forest Service Research Paper, INT-71.

Romme, W.H., Knight, D.H., Yavitt, J.B., 1986. Mountain pine beetle outbreaks in the Rocky Mountains: regulators of primary productivity? Am. Nat. 127, 484-494.

Rudinsky, J.A., Morgan, M.E., Libbey, L.M., Putnam, T.B., 1974. Antiaggreative-rivalry pheromone of the mountain pine beetle, and a new arrestant of the southern pine beetle. Environ. Entomol. 3, 90-98.

Ryker, L.C., Yandell, K.L., 1983. Effect of verbonone on aggregation of Dendroctonous ponderosae Hopkins (Coleoptera: Scolytidae) to synthetic attractant. Entomology $96,452-459$.

Safranyik, L., Silversides, R., McMullen, L.H., Linton, D.A., 1989. An empirical approach to modeling the local dispersal of the mountain pine beetle (Dendroctonus ponderosae Hopkins) in relation to sources of attraction, wind direction and speed. J. Appl. Entomol. 108, 498-511.

Schowalter, T.D., Coulson, R.N., Crossley, D.A., 1981. Role of southern pine beetle and fire in maitenance of structure and function of the southeastern coniferous forest. Environ. Entomol. 10, 821-825.
Smith, R.H., 1963. Toxicity of pine resin vapors to three species of Dendroctonus bark beetles. J. Econ. Entomol. 56, 827831 .

Smith, R.H., 1966. Resin quality as a factor in the resistance of pines to bark beetles. In: England, H.D., Gerhold, R.E., McDermot, R.E., Schreiner, E.J., Winieski, J.A. (Eds.), Proceedings of the NATO and National Science Foundation. Pergamon Press, Oxford, England, pp. 189-196.

Tennekes, H., Lumley, J.L., 1975. A First Course in Turbulence. MIT Press, Boston, MA.

Turchin, P., 1989. Population consequences of aggregative movement. J. Anim. Ecol. 58, 75-100.

Turchin, P., Simmons, G., 1997. Movements of animals in congregations: an Eulerian analysis of bark beetle swarming. In: Parrish, J.K., Hammer, W.M. (Eds.), Animal Groups in Three Dimensions, Cambridge University Press, USA.

Turchin, P., Simmons, G., 1998. Quantative Analysis of Movement: Measuring and Modeling Population Redistribution in Plants and Animals. Sinauer Associates, Sunderland, MA.

Turchin, P., Thoeny, W.T., 1993. Quantifying dispersal of southern pine beetles with mark-recapture experiments and a diffusion model. Ecol. Appl. 3, 187-198.

Wood, D.L., 1972. Selection and colonization of ponderosa pine by bark beetles. Symp. Roy. Entomol. Soc. London 6, 110-117. 